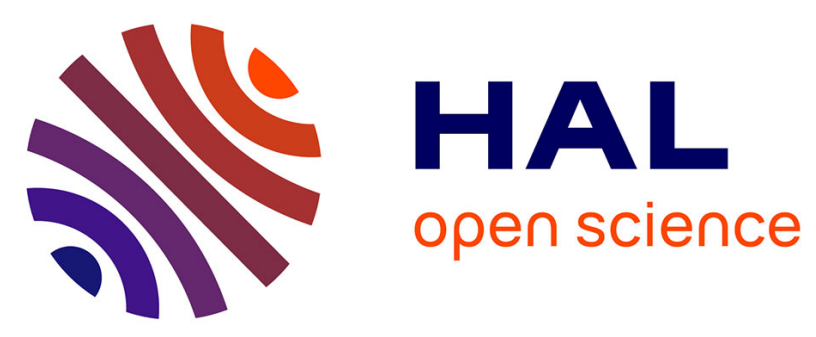

\title{
La séquence lacustre du maar d'Alleret (Massif Central, France) : téphrochronologie et évolution paléoenvironnementale en Europe occidentale au début du Pléistocène moyen
}

Jean-philippe Degeai, Jean-François Pastre, Agnès Gauthier, Vincent Robert, Sébastien Nomade, Viviane Bout-roumazeilles, Hervé Guillou

\section{To cite this version:}

Jean-philippe Degeai, Jean-François Pastre, Agnès Gauthier, Vincent Robert, Sébastien Nomade, et al.. La séquence lacustre du maar d'Alleret (Massif Central, France) : téphrochronologie et évolution paléoenvironnementale en Europe occidentale au début du Pléistocène moyen. Quaternaire, 2013, 24 (4), pp.443-459. 10.4000/quaternaire.6808 . hal-01483883

\section{HAL Id: hal-01483883 https://hal.science/hal-01483883}

Submitted on 26 Oct 2020

HAL is a multi-disciplinary open access archive for the deposit and dissemination of scientific research documents, whether they are published or not. The documents may come from teaching and research institutions in France or abroad, or from public or private research centers.
L'archive ouverte pluridisciplinaire HAL, est destinée au dépôt et à la diffusion de documents scientifiques de niveau recherche, publiés ou non, émanant des établissements d'enseignement et de recherche français ou étrangers, des laboratoires publics ou privés. 


\title{
LA SÉQUENCE LACUSTRE DU MAAR D'ALLERET (MASSIF CENTRAL, FRANCE) : TÉPHROCHRONOLOGIE ET ÉVOLUTION PALÉOENVIRONNEMENTALE EN EUROPE OCCIDENTALE AU DÉBUT DU PLÉISTOCÈNE MOYEN
}

\author{
Jean-Philippe DEGEAI ${ }^{1,2}$, Jean-François PASTRE², Agnès GAUTHIER ${ }^{2}$, \\ Vincent ROBERT ${ }^{2}$, Sébastien NOMADE ${ }^{3}$, Viviane BOUT-ROUMAZEILLES ${ }^{4}$ \\ \& Hervé GUILLOU ${ }^{3}$
}

\begin{abstract}
RÉSUMÉ
Le maar d'Alleret (Massif central, France) contient une séquence lacustre couvrant les stades isotopiques marins (SIM) 17 à 15. La partie étudiée, comprise entre 40,5 et $24,25 \mathrm{~m}$ de profondeur, enregistre la présence de téphras du Mont Dore (phase Sancy) qui permettent d'établir une téphrochronologie à partir de datations ${ }^{40} \mathrm{Ar} /{ }^{39} \mathrm{Ar}$ laser comprises entre $683 \pm 5$ et $718 \pm 6 \mathrm{ka}$. Les analyses géochimiques et minéralogiques de ces téphras montrent une mésostase vitreuse à composition trachytique à rhyolitique. Des analyses sédimentologiques (granulométrie laser, susceptibilité magnétique, carbone et azote total) permettent de proposer une reconstitution des dynamiques morphosédimentaires et de comprendre la géodynamique externe dans le bassin versant pendant la phase lacustre. Une étude palynologique complète cette étude et apporte des informations sur la dynamique de la végétation autour du maar. Dans la partie inférieure de la séquence, le développement successif de forêts riparienne, caducifoliée, puis montagnarde est caractéristique d'un interglaciaire, attribué au SIM 17. Cet interglaciaire est suivi par une phase glaciaire corrélée au stade SIM 16. Le début de cette période glaciaire montre dans la séquence d'Alleret une grande variabilité cyclique, avec la présence de trois cycles stade/interstade. La comparaison avec les données paléoenvironnementales issues de forages glaciels, marins et continentaux montrent une bonne adéquation avec le cadre chronostratigraphique de cette partie de la séquence d'Alleret et confirme le remarquable intérêt des maars du Massif central comme enregistreurs des variations climatiques et environnementales en milieu continental au cours du Quaternaire.
\end{abstract}

Mots-clés : paléoenvironnement, téphrochronologie, séquence lacustre, cratère de maar, Pléistocène moyen, Massif central

\section{ABSTRACT}

THE LACUSTRINE SEQUENCE OF THE ALLERET MAAR (MASSIF CENTRAL, FRANCE): TEPHROCHRONOLOGY AND PALAEOENVIRONNMENTAL EVOLUTION IN WESTERN EUROPE DURING THE EARLY MIDDLE PLEISTOCENE

The Alleret maar (Massif Central, France) is filled by a lacustrine sequence covering the marine isotopic stages (MIS) 17 to 15. In the studied part located between 24.25 and $40.5 \mathrm{~m}$ depth, the record of tephras from the Mont Dore (Sancy phase) allows us to establish a tephrochronology based on ${ }^{40} \mathrm{Ar} /{ }^{39} \mathrm{Ar}$ laser dating with ages between $683 \pm 5$ and $718 \pm 6 \mathrm{ka}$. The geochemical and mineralogical analyses of these tephras show a trachytic to rhyolitic glass composition. Sedimentological analyses (laser grain-size magnetic susceptibility, total carbon and nitrogen) allow us to propose a reconstruction of the morphosedimentary dynamics and to understand the external geodynamics in the watershed during the lacustrine phase. Palynological study achieves to reconstruct vegetation changes around the Alleret maar. In the lower part of the sequence, the succession of riparian, deciduous, and finally coniferous mountanous forests is representative of an interglacial period, which is attributed to the MIS 17. This interglacial is followed by the MIS 16 glacial period, which shows in the Alleret sequence a great cyclic variability with the presence of three stadial/interstadial cycles. Comparisons with ice, marine, and continental palaeoenvironmental and climatic records show a good agreement with the chronostratigraphical framework of this part of the Alleret sequence. Consequently, it confirms the great interest for the maars of the French Massif Central as markers of the environmental evolution and climate changes in continental domain during the Quaternary.

Keywords: palaeoenvironnment, tephrochronology, lacustrine sequence, maar crater, Middle Pleistocene, Massif Central

\footnotetext{
${ }^{1}$ Archéologie des Sociétés Méditerranéennes UMR 5140 CNRS et Université Montpellier 3, 390 route de Pérols, FR-34970 LATTES. Courriel : jean-philippe.degeai@cnrs.fr

${ }^{2}$ Laboratoire de Géographie Physique, Environnements Quaternaires et Actuels UMR 8591 CNRS et Universités Paris 1 et Paris 12, 1 place Aristide Briand, FR-92195 MEUDON CEDEX. Courriels : jean-françois.pastre@cnrs-bellevue.fr, agnes.gauthier@cnrs-bellevue.fr, vincent. robert@cnrs-bellevue.fr

${ }^{3}$ Laboratoire des Sciences du Climat et de l'Environnement, IPSL, CEA-CNRS et Université de Versailles Saint-Quentin, avenue de la Terrasse, FR-91198 GIF-SUR-YVETTE CEDEX. Courriels : sebastien.nomade@1sce.ipsl.fr, herve.guillou@1sce.ipsl.fr

${ }^{4}$ Géosystèmes UMR 8157 CNRS et Université Lille 1, bâtiment SN5, Cité Scientifique, FR-59655 VILLENEUVE D’ASCQ CEDEX. Courriel : viviane.bout@univ-lille1.fr
} 


\section{1 - INTRODUCTION}

La « Mid-Pleistocene Transition » est une période majeure de l'histoire du Quaternaire, marquée par de profonds bouleversements climatiques et atmosphériques à l'échelle planétaire comme le montrent les enregistrements marins et glaciels, avec notamment la durée des cycles climatiques passant de 41 à $100 \mathrm{ka}$ (Zachos et al., 2001 ; Ruddiman, 2003 ; Head \& Gibbard, 2005 ; Maslin \& Ridgwell, 2005 ; Clark et al., 2006 ; Berger \& Loutre, 2010). Des modifications régionales dans les faunes (Lourens et al., 2004 ; Maiorano \& Marino, 2004 ; Palombo et al., 2005), les flores (Tzedakis et al., 2006 ; Popescu et al., 2010), les rythmes de sédimentation et d'incision (Dodonov, 2005 ; Antoine et al., 2007 ; Bridgland \& Westaway, 2008 ; Gibbard \& Lewin, 2009) ou bien encore les déplacements de populations (Dennell, 2003) sont également observés durant cette période.

Les cratères de maar contiennent souvent de longues séquences sédimentaires ayant enregistré les évolutions paléoenvironnementales continentales en continu sur des périodes de plusieurs dizaines voire centaines de milliers d'années. Dans le Massif central, les maars du Velay et d'Auvergne offrent des séquences majeures pour reconstituer l'évolution de la végétation et du climat durant les 400 derniers millénaires, et servent ainsi de référence à l'échelle de l'Europe occidentale (Reille \& de Beaulieu, 1990, 1995 ; de Beaulieu \& Reille, 1992 ; Reille et al., 1998, 2000 ; de Beaulieu et al., 2001). Ces maars ont aussi permis d'étudier le paléomagnétisme du dernier cycle climatique (Thouveny et al., 1990, 1994), ou bien encore de reconstituer les vitesses de sédimentation et les rythmes d'érosion des sols depuis l'Eemien (Truze \& Kelts, 1993 ; Degeai \& Pastre, 2009).

Une première campagne de carottage réalisée en 2000 dans le remplissage sédimentaire du maar d'Alleret a permis d'échantillonner les quinze mètres supérieurs de cette séquence et de l'attribuer au SIM 15 (Pastre et al., 2007). En 2005, une seconde campagne de carottage a permis de traverser la totalité du remplissage intracratérique dans lequel une douzaine de téphras ont été retrouvés. La position géographique de ce maar au sud-est du Mont Dore permet d'enregistrer les téphras des épisodes éruptifs du Sancy qui offrent un important potentiel chronologique. La datation de ces téphras peut permettre d'établir une téphrochronologie pour la période du début du Pléistocène moyen, durant la seconde phase de la « Mid-Pleistocene Transition » entre 725 et 500 ka (Mudelsee \& Stattegger, 1997 ; Maslin \& Ridgwell, 2005).

Une étude pluri-indicateurs des seize mètres de la partie inférieure de la séquence du maar d'Alleret a été effectuée afin de proposer une reconstitution des dynamiques morphosédimentaires et paléoenvironnementales dans le bassin versant au début du Pléistocène moyen. Les résultats sont ensuite confrontés aux données paléobioclimatiques d'autres sites de référence (Benthic Stack LR04, EPICA Dome C, ODP982, Lac Baïkal, Tenaghi Philippon) afin d'apprécier la pertinence des données obtenues en milieu continental au maar d'Alleret.

\section{2 - CONTEXTE GÉOLOGIQUE}

Les cratères de maar correspondent à des reliefs volcaniques résultant d'une éruption phréatomagmatique, c'est-à-dire de l'interaction explosive entre un magma ascendant et une nappe phréatique (Lorenz, 1973, 1986). Ces cratères présentent des cavités relativement profondes dont le ratio profondeur/diamètre est en général de $1 / 5$ dans des roches mécaniquement résistantes à l'érosion (Lorenz, 1986 ; Degeai, 2004a). Lorsque l'évolution post-phréatomagmatique du cratère n'est pas d'origine volcanique et selon le niveau de la nappe phréatique, un lac de cratère peut s'installer et engendrer un remplissage sédimentaire lacustre post-éruptif progressif de la dépression cratérique, comme dans le cas du maar d'Alleret (Degeai, 2004b ; Pastre et al., 2007).

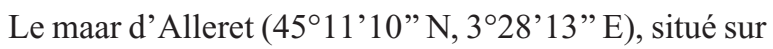
la commune de Saint-Privat-du-Dragon en Haute-Loire, constitue un vaste cratère d'explosion phréatomagmatique de $1500 \mathrm{~m}$ de diamètre (fig. 1). Il se situe au nord de la province basaltique du Devès, $13 \mathrm{~km}$ au sud-est de Brioude, en zone de moyenne montagne tempérée, à une altitude comprise entre 600 et $750 \mathrm{~m}$. Il recoupe les gneiss leptyno-amphibolitiques de la série métamorphique du Haut Allier à proximité de la limite occidentale du graben oligocène de Paulhaguet. Ce maar est bordé au nord-est par des scories stromboliennes qui représentent vraisemblablement la partie externe d'un cône strombolien partiellement détruit par l'ouverture du cratère de maar lors de l'éruption phréatomagmatique. Les dépôts phréatomagmatiques émis par cette éruption constituent un bourrelet en forme de croissant au sud du cratère, probablement plus étendu à l'origine, mais en partie érodé par la morphogenèse post-éruptive. Une intrusion de lave basique affleurant sur la paroi occidentale du cratère a été datée par K/Ar à $760 \mathrm{ka}$ (Fouris, 1989), et témoigne donc de l'âge postérieur au Pléistocène inférieur du maar.

L'étude morphosédimentaire, paléoenvironnementale et téphrochronologique de ce remplissage sédimentaire intracratérique lacustre a été rendue possible grâce à la réalisation de plusieurs carottages. La présente étude s'intéresse plus particulièrement au carottage $\mathrm{AL} / \mathrm{C} 3$, le plus profond, situé dans la partie centrale de l'ancienne plaine sous-lacustre du cratère. Ce carottage de 40,60 m de profondeur a atteint la base du remplissage sédimentaire post-éruptif et les dépôts phréatomagmatiques intracratériques situés à la partie sommitale du diatrème.

\section{3 - MATÉRIEL ET MÉTHODES}

\section{1 - LE CAROTTAGE AL/C3}

Le carottage $\mathrm{AL} / \mathrm{C} 3\left(45^{\circ} 11^{\prime} 11^{\prime \prime} \mathrm{N}, 3^{\circ} 28^{\prime} 10^{\prime \prime} \mathrm{E}\right.$, $\mathrm{Z}=607 \mathrm{~m}$ ) a été réalisé par forage tubé en fonçage et en roto-injection à circulation directe à l'aide de la sondeuse Bonne-Espérance du BRGM. Les carottes de sédiments ont été prélevées avec un carottier Mazier, dont la tête est munie d'un système de trousse coupante rétractable montée sur ressorts pour le prélèvement des sédiments 


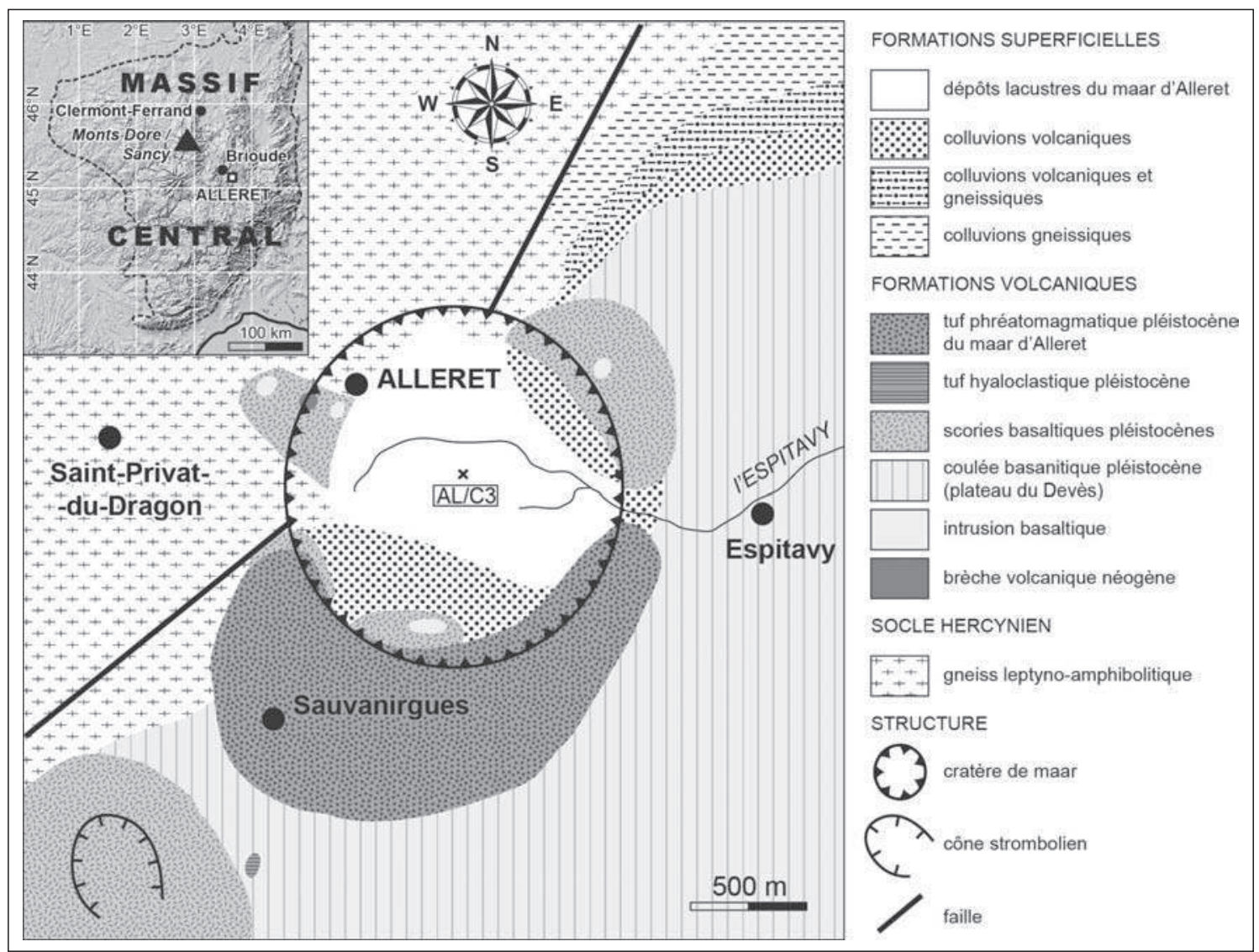

Fig. 1 : Carte géologique synthétique du maar d'Alleret et localisation du carottage AL/C3.

Fig. 1: Synthetic geological map of the Alleret maar and location of the AL/C3 core.

et d'une couronne rotative en carbure de tungstène pour l'évacuation des débris de forage (cuttings). L'avantage de ce système est de pouvoir traverser et prélever des terrains présentant une alternance de sédiments plus ou moins durs, comme ceci peut être le cas au maar d'Alleret (Pastre et al., 2007).

Toutefois, l'injection d'eau à travers le train de tige pour permettre l'avancement et l'évacuation des débris de forage peut entraîner un lessivage des formations peu consolidées tel que les sables. Ce phénomène explique très vraisemblablement la présence de plusieurs lacunes sédimentaires dans le carottage $\mathrm{AL} / \mathrm{C} 3$, d'une épaisseur cumulée de 4,7 m dans la partie du sondage comprise entre 0 et 23,6 $\mathrm{m}$ de profondeur.

Pour compléter ces lacunes de carottage, un second forage de $30 \mathrm{~m}$ de profondeur $(\mathrm{AL} / \mathrm{C} 4)$ a été entrepris à une dizaine de mètres du précédent, en adaptant la technique de carottage et l'utilisation du carottier en fonction des terrains traversés. Ce second carottage a permis de combler les lacunes existantes et sera étudié dans un prochain temps, selon la même méthodologie que celle appliquée pour le carottage AL/C3.

La présente étude concerne donc la partie du sondage $\mathrm{AL} / \mathrm{C} 3$ comprise entre $-40,50$ et $-24,25 \mathrm{~m}$, pour laquelle la séquence sédimentaire a pu être prélevée en continu (fig. 2). La séquence concernée est essentiellement constituée de sédiments argileux à argilo-silteux, parfois laminés (surtout à la base entre $-40,5$ et $-39,3 \mathrm{~m}$ et entre $-35,5$ et $-33 \mathrm{~m})$. Dans ces niveaux argileux peuvent

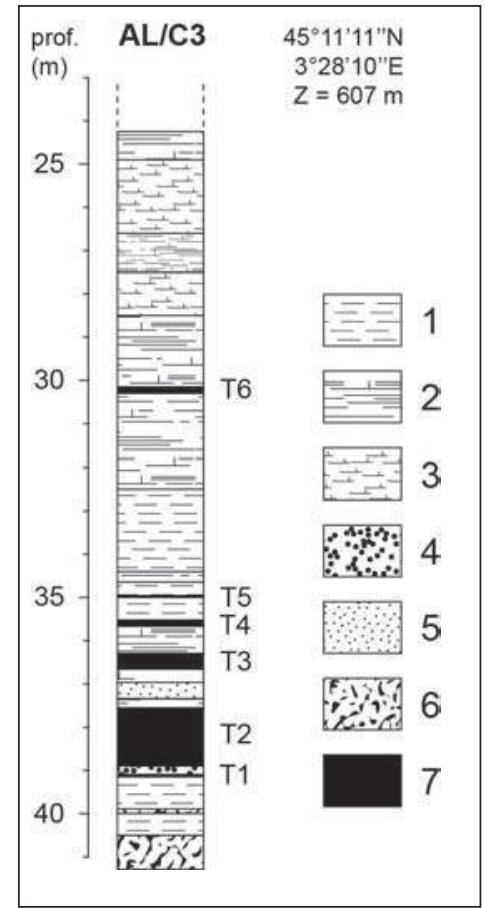

Fig. 2 : Log lithostratigraphique du carottage AL/C3 et position des téphras T1 à T6 dans la séquence sédimentaire du maar d'Alleret. $1 /$ argile laminée brune, 2/ argile grise à brune, 3/ silt argileux gris ou brun, 4/ argile sableuse, 5/ sable ponceux, 6/ graviers de basalte / tuf phréatomagmatique, $7 /$ téphra.

Fig. 2: Lithostratigraphical log of the AL/C3 core and position of the T1 to T6 tephras in the sedimentary sequence of the Alleret maar. 1/ brown laminated clay, 2/ brown to gray clay, 3/ gray or brown clayey silt, 4/ sandy clay, 5/ pumice sand, 6/ basalt gravel / phreatomagmatic tuff, 7/ tephra. 
s'interstratifier des couches silto-sableuses, sableuses, voire sablo-graveleuses comme à la base du sondage. Par ailleurs, on peut noter la présence de six téphras d'épaisseur centimétrique à métrique. Les téphras $\mathrm{T} 1$ à $\mathrm{T} 5$ sont localisés dans la partie inférieure du remplissage sédimentaire entre - 40,50 et $-35 \mathrm{~m}$. Le téphra T6 se situe un peu plus haut dans la séquence à - 30,20 m.

\section{2 - DATATIONS ${ }^{40} \mathrm{AR} /{ }^{39} \mathrm{AR}$ LASER}

Les datations ${ }^{40} \mathrm{Ar} /{ }^{39} \mathrm{Ar}$ ont été réalisées sur des grains de sanidine sodique non altérés d'une taille comprise entre 160 et $250 \mu \mathrm{m}$, légèrement décapés dans une solution d'acide fluorhydrique concentré à $5 \%$ pendant $5 \mathrm{mn}$ (Nomade et al., 2010). Un total de 25 à 30 grains a été chargé pour chaque échantillon dans des disques d'aluminium et irradiés $40 \mathrm{mn}$ dans un tube $\beta 1$ du réacteur OSIRIS (CEA Saclay). Le flux neutronique total reçu par les échantillons se situe entre 1,2 et $2,7 \times 10^{-14} \mathrm{n} . \mathrm{cm}^{-2}$. Après l'irradiation, chaque grain a été chauffé à une température modérée équivalente à $3 \%$ de la puissance du laser en utilisant un laser $\mathrm{CO}_{2}$ de $25 \mathrm{~W}$ (Synrad) afin de nettoyer le grain de toute contamination atmosphérique. Les isotopes de l'argon ont été analysés avec un spectromètre de masse VG5400 équipé d'un compteur d'ion unique (Balzers SEV 217 SEN). Un total de 19 à 20 grains a été analysé individuellement pour chaque échantillon. La précision de cette technique permet théoriquement de mesurer avec fiabilité des échantillons contenant moins de $1 \%$ de l'isotope ${ }^{40} \mathrm{Ar}$. Les taux de production nucléogénique utilisés pour corriger les isotopes de l'argon produits par le réacteur à partir des éléments du potassium et du calcium sont mentionnés dans Scaillet et al. (2008). Les âges ${ }^{40} \mathrm{Ar}{ }^{39} \mathrm{Ar}$ sont calculés relativement au standard ACs à 1,193 Ma (Nomade et al., 2005) en utilisant la constante de désintégration $\mathrm{du}^{40} \mathrm{~K}$ de Steiger \& Jäger (1977).

\section{3 - MINÉRALOGIE ET GÉOCHIMIE DES TÉPHRAS}

\subsection{1 - Géochimie des verres volcaniques et phéno- cristaux}

La composition chimique en éléments majeurs des verres volcaniques, des feldspaths et des magnétites a été déterminée par analyse à la microsonde électronique (EMP). Les analyses ont été réalisées sur la microsonde Cameca SX100 à quatre spectromètres WDX du service commun Camparis (Jussieu, CNRS). Après tamisage, des clastes d'environ $1 \mathrm{~mm}$ de diamètre ont été sélectionnés sous loupe binoculaire et montés en pastille dans de la résine époxy. Le polissage final de la pastille a été réalisé avec une émulsion de poudre de diamant d'une finesse de $1 \mu \mathrm{m}$. La surface des pastilles a été métallisée par un film de carbone afin d'assurer la conductivité des grains. La tension et l'intensité du faisceau électronique incident sont respectivement de $15 \mathrm{keV}$ et $4 \mathrm{nA}$. Le faisceau a été réglé sur une taille de $4 \mu \mathrm{m}$ et a été défocalisé pour l'analyse des verres afin de limiter la décroissance des éléments volatils tels que le sodium. Pour les feldspaths, la taille du faisceau était de $10 \mu \mathrm{m}$. Les éléments quantifiés sont $\mathrm{Na}, \mathrm{Mg}, \mathrm{Si}, \mathrm{Al}, \mathrm{K}, \mathrm{Ca}, \mathrm{Ti}, \mathrm{Fe}, \mathrm{Mn}$. La quantification des éléments mesurés est réalisée par référence à la composition chimique de minéraux standard. Il s'agit d'albite pour $\mathrm{Na}$, d'un diopside pour $\mathrm{Mg}$, $\mathrm{Si}$ et $\mathrm{Ca}$, d'une orthose pour $\mathrm{Al}$ et $\mathrm{K}$, d'un oxyde de titano-manganèse pour Ti et $\mathrm{Mn}$, et d'un grenat pour Fe. Les grains de ponces et les feldspaths de chaque téphra ont fait l'objet de 10 à 21 mesures. Pour les verres, la composition a été normalisée à $100 \%$ sur une base anhydre.

\subsection{2 - Détermination des cortèges de minéraux lourds}

L'analyse des minéraux lourds contenus dans les téphras a été réalisée sur la fraction sableuse $(50 \mu \mathrm{m}$ à $2 \mathrm{~mm}$ ) après séparation densimétrique par gravité d'environ $100 \mathrm{~g}$ de particules dans du bromoforme $(\mathrm{d}=2,82 \mathrm{et}$ $\left.\eta=2 \mathrm{mPa} \cdot \mathrm{s}^{-1}\right)$. La détermination des principales espèces minéralogiques a été effectuée à l'aide d'un microscope optique et d'une loupe binoculaire. Les comptages ont été effectués sur une population de 200 à 300 grains (Parfenoff et al., 1970).

\subsection{3 - Analyse texturale des ponces au MEB}

Pour chaque téphra du sondage $\mathrm{AL} / \mathrm{C} 3$, une dizaine de grains de ponce a été sélectionnée et montée sur un film carbone afin d'observer au MEB la texture de la phase vitreuse. La surface des grains a été métallisée sous un plasma argon pour obtenir un film d'une épaisseur de 40 à $70 \mathrm{~nm}$. Les paramètres du faisceau électronique ont été réglés à $10 \mathrm{keV}$ avec une intensité de courant comprise entre 0,1 et $1 \mu \mathrm{A}$.

\section{4 - SÉDIMENTOLOGIE DES DÉPÔTS LACUSTRES}

\subsection{1 - Granulométrie}

Des analyses granulométriques par diffraction laser ont été effectuées pour 319 échantillons (résolution verticale moyenne de $5 \mathrm{~cm}$ ), sur un granulomètre laser Coulter LS230, qui permet de mesurer la distribution granulométrique des sédiments selon 116 classes allant de $40 \mathrm{~nm}$ à $2 \mathrm{~mm}$ de diamètre particulaire. Un traitement à l'eau oxygénée à 130 volumes a été réalisé au préalable afin de détruire la matière organique. La prise d'essai varie de 2 à $10 \mathrm{~g}$ selon la granularité du sédiment. Les analyses ont été réalisées en voie liquide à l'aide du Fluid Module du granulomètre. La dispersion des échantillons a été assurée à l'aide d'un agent tensio-actif (hexamétaphosphate de sodium à $0,5 \%$ ), d'un agitateur mécanique réglé à $20 \mathrm{~Hz}$ pendant $15 \mathrm{mn}$, et d'un passage dans une cuve à ultrasons à $20 \mathrm{kHz}$ pendant $1 \mathrm{mn}$. Plusieurs aliquotes de 2 à $4 \mathrm{ml}$ ont été versées afin d'obtenir une concentration de particules suffisante dans le circuit hydraulique $\mathrm{du}$ granulomètre. Les taux d'obscuration sur la cellule de diffraction et la cellule PIDS (Polarization Intensity Differential Scattering) sont respectivement compris entre 8 et $12 \%$ et entre 45 et $65 \%$. Le modèle optique de 
Fraunhofer a été utilisé avec comme paramètre principal l'indice de réfraction du fluide dans lequel les particules sont en suspension $\left(\mathrm{n}_{\mathrm{w}}=1,332\right)$. Des macros en langage Visual Basic ont été utilisées afin d'interpoler les limites de classe et de calculer les paramètres granulométriques, avec des coupures argile/limon et limon/sable choisies respectivement à 6 et $63 \mu \mathrm{m}$ (diamètre équivalent laser).

La répétabilité des distributions granulométriques a été vérifiée sur 114 échantillons selon la norme ISO 13320-1, afin de s'assurer que l'erreur RSD sur 2 ou 3 mesures consécutives reste inférieure à $3 \%$ pour la médiane et $5 \%$ pour les déciles d10 et $\mathrm{d} 90$.

Les analyses de granulométrie laser ont été effectuées sur les particules inférieures à $1 \mathrm{~mm}$ de diamètre. Pour 45 échantillons plus grossiers, des analyses par tamisage ont été réalisées pour la fraction du sédiment comprise entre 1 et $5 \mathrm{~mm}$ sur 8 tamis $(1 ; 1,25 ; 1,6 ; 2 ; 2,5 ; 3,15$; $4 ; 5 \mathrm{~mm})$. Les deux distributions ont été combinées en pondérant chaque distribution (laser et tamis) par son poids relatif.

\subsection{2 - Susceptibilité magnétique}

La susceptibilité magnétique spécifique de masse a été mesurée avec un capteur à double fréquence Bartington MS2B. Les mesures ont été effectuées sur 319 échantillons en basse fréquence $(0,465 \mathrm{kHz})$ et en haute fréquence $(4,65 \mathrm{kHz})$ avec un champ magnétique appliqué de $250 \mu \mathrm{T}$. La fraction inférieure à $2 \mathrm{~mm}$ des sédiments, préalablement séchés et broyés, a été placée dans des pots calibrés d'un volume de $10 \mathrm{~cm}^{3}$. Le coefficient de dépendance à la fréquence de la susceptibilité magnétique a été mesuré afin de déterminer le pourcentage de particules superparamagnétiques qui donnent une information sur l'intensité des processus d'altération biochimique (Dearing et al., 1996a, 1996b). Les mesures ont été réalisées en unité SI et en gamme (range) 1 pour les valeurs supérieures à $100 \times 10^{-8} \mathrm{~m}^{3} \cdot \mathrm{kg}^{-1}$, et en gamme 0,1 pour les valeurs inférieures à $100 \times 10^{-8} \mathrm{~m}^{3} \cdot \mathrm{kg}^{-1}$.

\subsection{3 - Analyse élémentaire carbone/azote}

L'analyse du carbone total et de l'azote total a été réalisée sur un analyseur élémentaire FlashEA Thermo Fisher séries 1112, respectivement selon les normes NF ISO 10694 et NF ISO 13878. Les analyses ont été effectuées sur 72 échantillons avec une résolution verticale de $20 \mathrm{~cm}$. Les échantillons ont été préalablement séchés $24 \mathrm{~h}$ à $70^{\circ} \mathrm{C}$ avant d'être broyés et tamisés à $200 \mu \mathrm{m}$. Ils ont ensuite été pesés puis introduits dans une nacelle en étain. Ils subissent une combustion flash à $920^{\circ} \mathrm{C}$ sous oxygène gazeux $\left(\mathrm{O}_{2}\right)$. Les oxydes d'azote $\left(\mathrm{NO}_{\mathrm{x}}\right)$ produits sont réduits sous forme d'azote moléculaire $\left(\mathrm{N}_{2}\right)$ via un passage sur du cuivre métallique à $680^{\circ} \mathrm{C}$. Le $\mathrm{CO}_{2}$ et le $\mathrm{N}_{2}$ sont séparés sur une colonne chromatographique et les teneurs en carbone et azote sont mesurées par conductibilité thermique. Un étalonnage externe utilisant un standard carboné et azoté permet la quantification, avec une erreur relative inférieure à $5 \%$ sur les gammes de valeurs mesurées.

\section{5 - PALYNOLOGIE}

Les analyses palynologiques du sondage AL/C3 ont porté sur 123 échantillons (1 seul s'est révélé stérile) issus de niveaux argileux ou argilo-silteux. La préparation chimique classique (Faegri \& Iversen, 1989) a été adaptée aux argiles organiques très compactes, de manière à obtenir des résultats satisfaisants. Le traitement a comporté les étapes suivantes : $\mathrm{KOH} 10 \%$, acétolyse, $\mathrm{HF} 70 \%, \mathrm{HCl} 50 \%, \mathrm{KOH} 10 \%$, dilution dans la glycérine bi-distillée, mesure du culot, montage entre lame et lamelle de $40 \mu \mathrm{l}$. L'analyse microscopique a été réalisée à 1 'objectif $\times 25$ ou $\times 50$ et toutes les déterminations ont été faites à l'objectif x100. Pour chaque échantillon, un minimum de 400 grains de pollen dont au moins 150 en dehors du taxon dominant a été compté. Ce nombre de grains se situe entre 383 et 1051 grains (moyenne de 530 grains). Le nombre de taxons polliniques déterminés pour chaque échantillon se situe entre 21 et 42 (moyenne de 30 taxons). Les pourcentages polliniques ont été calculés à partir de la somme pollinique de base excluant Pinus, Ptéridophytes, Indéterminés et Indéterminables. Pinus a été exclu en raison de sa surreprésentation résultant de son taux de production élevé et de sa très bonne flottaison dans l'air et l'eau (Huntley \& Birks, 1983 ; Guérin, 1993). Les pourcentages de Pinus ont été calculés à partir de la somme pollinique de base plus Pinus.

\section{4 - RÉSULTATS}

\section{1 - CHRONOLOGIE}

Trois datations ${ }^{40} \mathrm{Ar} /{ }^{39} \mathrm{Ar}$ laser ont été effectuées pour les téphras T6, T3 et T1, situés respectivement à 30,2, $36,9 \mathrm{~m}$ et $39,6 \mathrm{~m}$ de profondeur (fig. 3). Les trois autres téphras T2, T4 et T5 feront l'objet de futures datations. Le spectre de probabilité d'âge pour le téphra T6 affiche une distribution unimodale avec une légère dissymétrie probablement due à un excès de la composante ${ }^{40} \mathrm{Ar}$ dans certains grains. En excluant deux grains afin d'obtenir une erreur MSWD (mean-square weighted deviation) acceptable de 1,2 , un âge de $683 \pm 5$ ka $(2 \sigma, \mathrm{n}=17 / 19)$ est déterminé. Pour le téphra T3, le spectre de probabilité d'âge montre une distribution unimodale à partir de laquelle un âge d'éruption de $713 \pm 5 \mathrm{ka}(2 \sigma)$ est calculé ( $n=19 / 19$, MSWD de 0,7). Concernant le téphra T1, le spectre de probabilité d'âge est de nouveau unimodal mais présente une plus large dispersion comparée aux résultats obtenus pour les deux téphras précédents. Cette dispersion apparaît dans le spectre comme une forte dissymétrie vers les âges plus anciens. Si les 20 cristaux sont pris en compte dans le calcul de l'âge, l'erreur MSWD augmente à 4,4, suggérant une dispersion excessive. L'erreur MSWD diminue à une valeur plus raisonnable de 3,1 si les cristaux les plus vieux sont exclus de la moyenne pondérée. L'exclusion d'un nombre plus important de cristaux afin d'obtenir une valeur MSWD inférieure à 2 devrait requérir l'élimination d'environ 

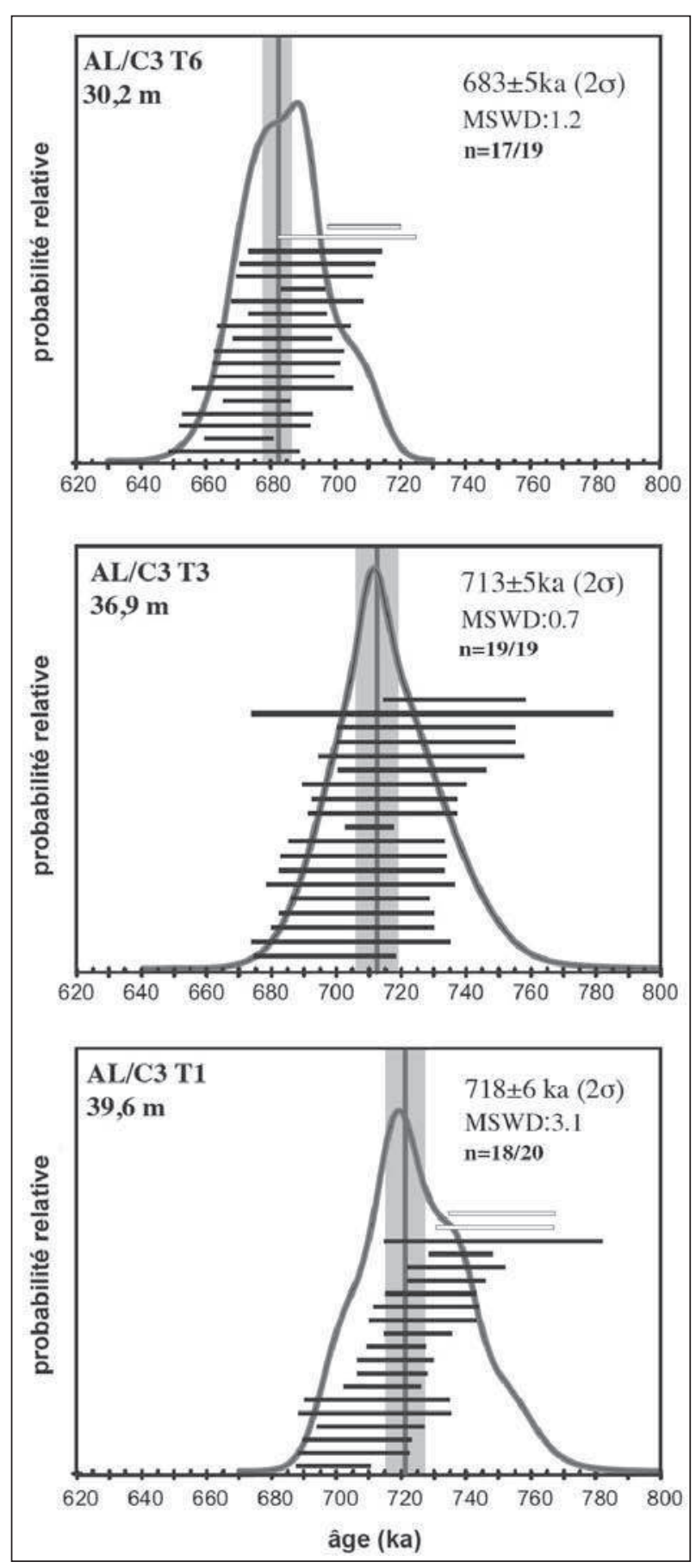

Fig. 3 : Spectres de probabilité d'âge et datations ${ }^{40} \mathrm{Ar} /{ }^{39} \mathrm{Ar}$ laser des téphras T1, T3 et T6 de la séquence $\mathrm{AL} / \mathrm{C} 3$ du maar d'Alleret. Les barres noires et grises représentent respectivement les grains retenus et rejetés.

Fig. 3: Age probability spectra and ${ }^{40} \mathrm{Ar}{ }^{{ }^{39}} \mathrm{Ar}$ laser ages of $T 1, T 3$ and T6 tephras from the AL/C3 sequence of the Alleret maar. The black and grey bars represent respectively kept and rejected grains.

$40 \%$ des cristaux. La plus forte dispersion peut être liée à un mélange de cristaux avec et sans un léger excès de la composante ${ }^{40} \mathrm{Ar}$ (Nomade et al., 2010). Le meilleur âge pour ce téphra serait de $718 \pm 6 \mathrm{ka}(2 \sigma, \mathrm{n}=18 / 20)$.

\section{2 - TÉPHROSTRATIGRAPHIE}

Les téphras du carottage $\mathrm{AL} / \mathrm{C} 3$ correspondent à des retombées cendreuses à micro-ponces épaisses de 1 à $38 \mathrm{~cm}$, à l'exception du téphra T2, qui atteint $140 \mathrm{~cm}$ d'épaisseur, avec des clastes de ponces et des xénolites de basalte pouvant dépasser $1 \mathrm{~cm}$ de diamètre. L'analyse microchimique des verres (tab. 1) et des feldspaths et la minéralogie des échantillons (minéralogie globale, minéraux lourds) permettent de caractériser et de distinguer les différents niveaux.

Les verres (microponces) sont conservés dans les téphras T2 à $\mathrm{T} 6$ et altérés dans les autres niveaux de retombées. Avec des valeurs de $\mathrm{SiO}_{2}$ comprises entre 63 et $66 \%$ et celles des alcalins $\mathrm{Na}_{2} \mathrm{O}+\mathrm{K}_{2} \mathrm{O}$ comprises entre 12,3 et $13,7 \%$, les verres des téphras T2, T3, T4 et T5 sont trachytiques (diagramme TAS, fig. 4). Le verre du téphra T6, qui comporte 69,9-70,5\% de $\mathrm{SiO}_{2}$ et $11-11,3 \%$ d'alcalins est plus différencié, et se range dans le champ des rhyolites. Toutefois, ces compositions n'étant pas représentatives de la roche totale, il faut considérer que ces produits sont en réalité légèrement moins différenciés et que le téphra T6 puisse être également trachytique. Cependant, les observations au MEB effectuées sur les micro-ponces de ce téphra montrent une mésostase vitreuse à texture fibreuse, qui se distingue nettement de celle à texture vacuolaire et «moussue » des autres téphras (fig. 5).

Les pourcentages des différents oxydes varient selon les téphras et permettent d'établir une discrimination géochimique. Le diagramme $\mathrm{FeOt} / \mathrm{CaO}$ illustre cette discrimination réalisable à partir des éléments majeurs $\mathrm{Fe}$ et Ca (fig. 4). Les téphras T2 et T3 montrent des teneurs pondérales intermédiaires en oxydes de fer total et de calcium, avec des valeurs comprises respectivement entre 1,6 et $2,2 \%$ et entre 0,8 et $1,1 \%$. Les téphras T4 et T5 présentent une composition plus riche en fer (FeOt entre $1,9$ et $2,5 \%)$ et en calcium ( $\mathrm{CaO}$ entre 1,1 et $1,5 \%)$, à l'inverse du téphra T6 (FeOt entre 1 et $1,6 \%$ et $\mathrm{CaO}$ entre 0,5 et $0,75 \%$ ).

Cette séparation en trois groupes se retrouve sur les diagrammes bi-variés opposant la teneur pondérale en silice à celle des alcalins $\left(\mathrm{Na}_{2} \mathrm{O}\right.$ et $\left.\mathrm{K}_{2} \mathrm{O}\right)$, des oxydes d'aluminium, de magnésium, de calcium et de fer total (fig. 4). Les téphras T2 et T3 présentent dans tous les cas une composition moyenne, tandis que les téphras T4 et T5, moins acides et plus calco-alcalins, sont plus riches en magnésium, en aluminium et en fer, à l'inverse du téphra T6. Le diagramme $\mathrm{SiO}_{2} / \mathrm{MgO}$ permet par ailleurs d'opérer une discrimination entre les téphras T4 et T5, le premier montrant dans l'ensemble une teneur légèrement plus élevée en magnésium le plaçant dans une position intermédiaire entre le groupe de téphras T2/T3 et le téphra T5. Le diagramme $\mathrm{SiO}_{2} / \mathrm{TiO}_{2}$ permet de bien distinguer les cinq téphras analysés (fig. 4). Le titane est un élément discriminant au sein des groupes T2/T3 et T4/ $\mathrm{T} 5$, les téphras $\mathrm{T} 2$ et $\mathrm{T} 4$ présentant une teneur pondérale en $\mathrm{TiO}_{2}$ plus forte que celle des téphras $\mathrm{T} 3$ et T5.

Les feldspaths constituent les phases minérales prépondérantes. Ils ont fait l'objet d'analyses microchimiques systématiques dans les différents niveaux. Les variations des pourcentages classiques d'anorthite (An), albite (Ab) et orthose (Or) sont caractéristiques de chaque unité. Les résultats étant trop nombreux pour être récapitulés dans un diagramme triangulaire, les compositions sont résu- 


\begin{tabular}{|l|cc|cc|cc|cc|cc|}
\hline $\begin{array}{r}\text { Téphra } \\
n\end{array}=$ & \multicolumn{2}{|c|}{ AL/C3 T2 } & \multicolumn{2}{c|}{ AL/C3 T3 } & \multicolumn{2}{c|}{ AL/C3 T4 } & \multicolumn{2}{c|}{ AL/C3 T5 } & \multicolumn{2}{c|}{ AL/C3 T6 } \\
& \% p.o. & $1 \sigma$ & \% p.o. & $1 \sigma$ & $\%$ p.o. & $1 \sigma$ & $\%$ p.o. & $1 \sigma$ & $\%$ p.o. & $1 \sigma$ \\
\hline $\mathrm{Na}_{2} \mathrm{O}$ & 6,41 & 0,14 & 6,52 & 0,29 & 6,68 & 0,25 & 6,95 & 0,24 & 5,67 & 0,16 \\
$\mathrm{MgO}$ & 0,24 & 0,03 & 0,25 & 0,02 & 0,30 & 0,03 & 0,34 & 0,01 & 0,13 & 0,02 \\
$\mathrm{SiO}_{2}$ & 65,84 & 0,31 & 65,89 & 0,22 & 63,87 & 0,36 & 63,75 & 0,30 & 70,47 & 0,25 \\
$\mathrm{Al}_{2} \mathrm{O}_{3}$ & 17,24 & 0,23 & 17,35 & 0,13 & 18,16 & 0,18 & 18,38 & 0,19 & 15,77 & 0,26 \\
$\mathrm{~K}_{2} \mathrm{O}$ & 6,22 & 0,25 & 6,35 & 0,28 & 6,43 & 0,23 & 6,37 & 0,20 & 5,55 & 0,20 \\
$\mathrm{CaO}$ & 0,91 & 0,05 & 0,98 & 0,05 & 1,25 & 0,07 & 1,17 & 0,05 & 0,63 & 0,05 \\
$\mathrm{TiO}_{2}$ & 1,12 & 0,17 & 0,52 & 0,16 & 1,01 & 0,38 & 0,63 & 0,10 & 0,27 & 0,11 \\
$\mathrm{FeO}$ & 1,85 & 0,14 & 1,96 & 0,07 & 2,15 & 0,14 & 2,23 & 0,12 & 1,36 & 0,11 \\
$\mathrm{MnO}$ & 0,17 & 0,03 & 0,17 & 0,08 & 0,16 & 0,05 & 0,18 & 0,06 & 0,14 & 0,07 \\
\hline
\end{tabular}

Tab. 1 : Composition chimique des verres volcaniques des téphras de la séquence AL/C3 du maar d'Alleret. Résultats normalisés à $100 \%$ sur une base anhydre.

Tab. 1: Geochemical composition of volcanic glass of the tephras from the AL/C3 sequence of the Alleret maar. Results normalized to $100 \%$ on an anhydrous basis.

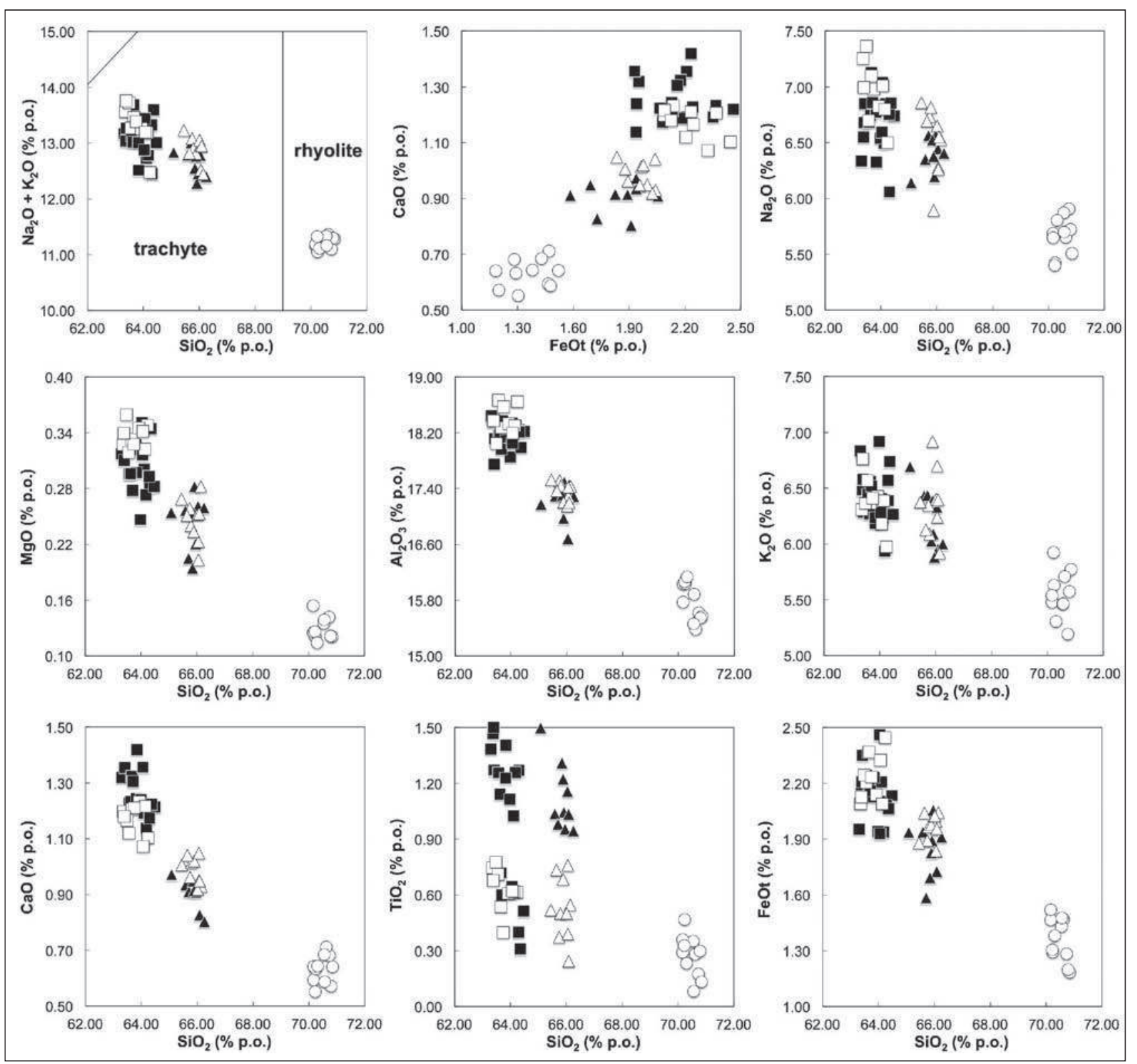

Fig. 4 : Diagrammes bi-variés des compositions en poids d'oxyde des verres volcaniques des téphras T2 à T6 du carottage AL/C3 du maar d'Alleret.

Triangle noir : téphra T2, triangle blanc : téphra T3, carré noir : téphra T4, carré blanc : téphra T5, rond blanc : téphra T6, p.o. : poids d'oxyde.

Fig. 4: Bivariate oxide-oxide wt\% plots for the volcanic glass in the T2 to T6 tephras from the Alleret maar AL/C3 core. Black triangle: T2 tephra, white triangle: T3 tephra, black square: T4 tephra, white square: T5 tephra, white circle: T6 tephra, p.o.: oxide weight. 


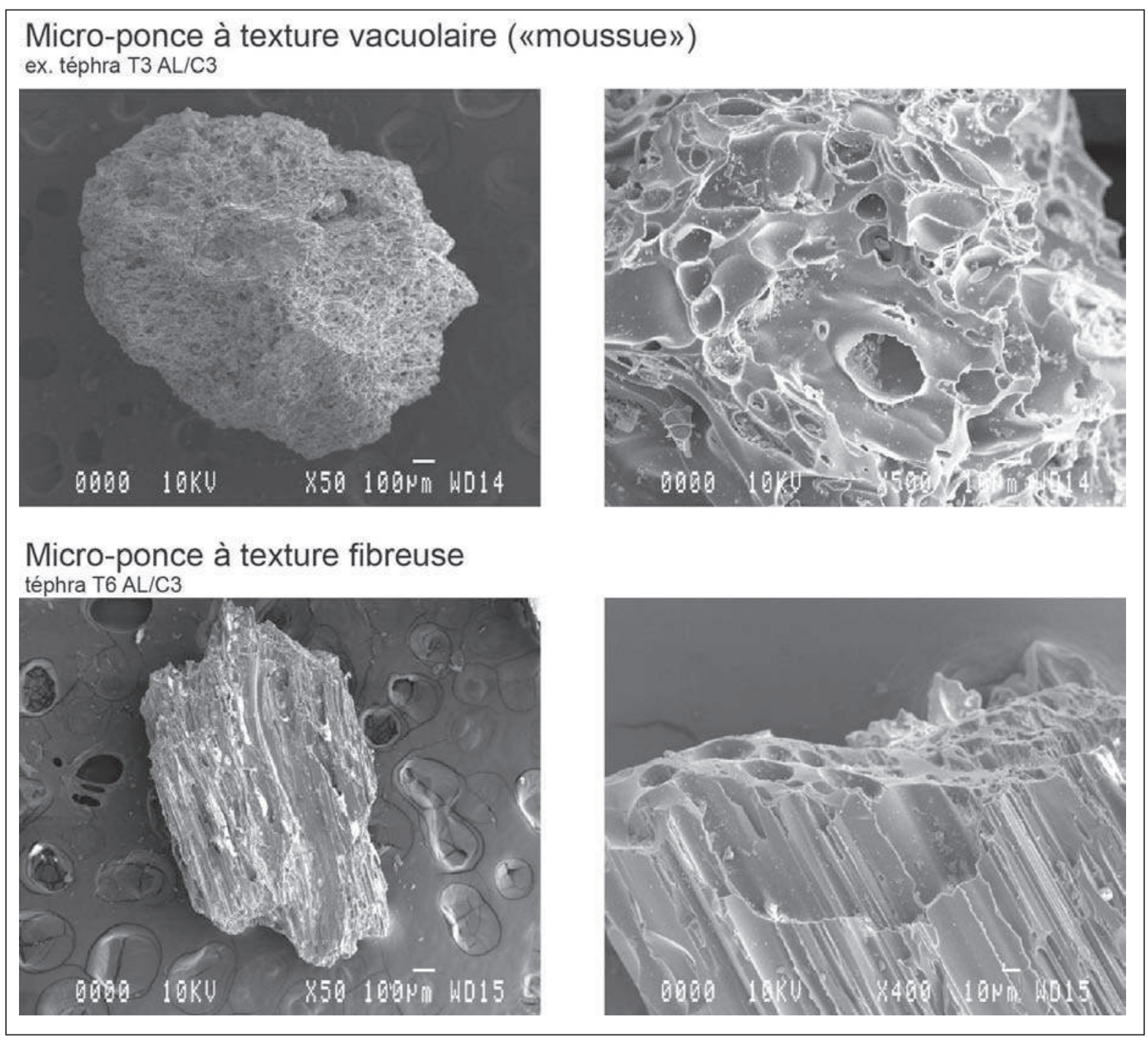

Fig. 5 : Texture des grains de ponce des téphras du carottage AL/C3 du maar d'Alleret.

Fig. 5: Texture of pumice grains of the tephras from the $A L / C 3$ core from the Alleret maar.

mées dans le tableau 2. Les feldspaths des téphras T1 à T3 sont tous des feldspaths alcalins avec des compositions d'anorthose ou de sanidine sodique. Les feldspaths des téphras T4 et T5 comportent à la fois des feldspaths alcalins (anorthose et sanidine sodique) et des plagioclases (andésine et oligoclase potassique + rares labradors et bytownites correspondant probablement à des xénolites). Les feldspaths du téphra T6 sont dominés par les feldspaths alcalins auxquels s'ajoutent quelques oligoclases.

Les autres silicates et l'apatite sont regroupés dans les cortèges de minéraux lourds rapportés à $100 \%$ (fig. 6). Ces cortèges sont dominés par le clinopyroxène vert (diopside, 54 à $96 \%$ ), qui constitue un minéral caractéristique des produits trachytiques du Sancy. Les amphiboles brunes sont généralement absentes ou peu représentées. La titanite (sphène), toujours présente, est soit rare (ex. $\mathrm{T} 5=3,3 \%)$, soit abondante $(\mathrm{T} 6=41,9 \%)$. Les cortèges sont complétés par de l'apatite, qui reste relativement rare (moins de $5 \%$ ). Les oxydes de Fe-Ti comportent de la titanomagnétite, de composition variable (majoritaire), et de l'ilménite (minoritaire).

\section{3 - SÉDIMENTOLOGIE}

Les données de granulométrie montrent entre $-40,50$ et $-34,40 \mathrm{~m}$ (unités U1 à U2a) des niveaux généralement riches en particules fines argileuses et limoneuses, avec des valeurs moyennes respectives de 30 et $60 \%$, mais aussi la présence de plusieurs passées sableuses, avec des enrichissements ponctuels de sable à $80 \%$ (fig. 7). Entre -34,40 et - 32,5 m (U2b et U2c), les sédiments présentent une texture granulométrique globalement semblable à celle du niveau précédent mais sans les passées sableuses, témoignant vraisemblablement d'une dynamique morphogénique moins énergique se traduisant par une sédimentation plus fine. De - 32,50 à -30,15 m (unité U3), la hausse de la fraction sableuse de 5 à $40 \%$, montre vraisemblablement une reprise des processus détritiques mobilisant la charge particulaire grossière à la surface du bassin versant. De - 30,15 à -24,25 m (U4), les niveaux de sables sont généralement élevés, avec des maximas atteignant $70 \%$ vers $28 \mathrm{~m}$ de profondeur. Dans le détail, la granulométrie des sables montre toutefois une variabi- 


\begin{tabular}{|c|c|c|c|c|c|}
\hline $\mathrm{N}^{\circ}$ & $\begin{array}{l}\text { Composition des } \\
\text { feldspaths alcalins }\end{array}$ & $\begin{array}{l}\text { Nature des feldspaths } \\
\text { alcalins }\end{array}$ & $\begin{array}{l}\text { Composition des } \\
\text { plagioclases }\end{array}$ & $\begin{array}{l}\text { Nature des } \\
\text { plagioclases }\end{array}$ & Observations \\
\hline $\begin{array}{l}\mathrm{T} 1 \\
(14)\end{array}$ & $\begin{array}{lll}\mathrm{Or}_{25} & \mathrm{Ab}_{66} & \mathrm{An}_{9} \text { à } \\
\mathrm{Or}_{45} & \mathrm{Ab}_{52} \mathrm{An}_{3}\end{array}$ & $\begin{array}{l}\text { anorthose/sanidine } \\
\text { sodique }\end{array}$ & & & \\
\hline $\begin{array}{l}\mathrm{T} 2 \\
(16)\end{array}$ & $\begin{array}{l}\mathrm{Or}_{23} \mathrm{Ab}_{66} \mathrm{An}_{11} \mathrm{a} \\
\mathrm{Or}_{47} \mathrm{Ab}_{50} \mathrm{An}_{3}\end{array}$ & $\begin{array}{l}\text { anorthose/sanidine } \\
\text { sodique }\end{array}$ & & & \\
\hline $\begin{array}{l}\mathrm{T} 3 \\
(17)\end{array}$ & $\begin{array}{l}\mathrm{Or}_{24} \mathrm{Ab}_{66} \mathrm{An}_{10} \text { à } \\
\mathrm{Or}_{45} \mathrm{Ab}_{52} \mathrm{An}_{3}\end{array}$ & $\begin{array}{l}\text { anorthose/sanidine } \\
\text { sodique }\end{array}$ & & & \\
\hline $\begin{array}{l}\mathrm{T} 4 \\
(16)\end{array}$ & $\begin{array}{lll}\mathrm{Or}_{29} & \mathrm{Ab}_{61} & \mathrm{An}_{10} \\
\mathrm{Or}_{37} & \mathrm{Ab}_{57} & \mathrm{An}_{6}\end{array}$ & $\begin{array}{l}\text { anorthose/sanidine } \\
\text { sodique }\end{array}$ & $\begin{array}{l}\mathrm{Or}_{5} \mathrm{Ab}_{59} \mathrm{An}_{36} \mathrm{à} \\
\mathrm{Or}_{12} \mathrm{Ab}_{67} \mathrm{An}_{21}\end{array}$ & $\begin{array}{l}\text { andésine/oligoclase } \\
\text { potassique }\end{array}$ & $\begin{array}{l}\text { rares labradors et } \\
\text { bytownites }\end{array}$ \\
\hline $\begin{array}{l}\text { T5 } \\
(21)\end{array}$ & $\begin{array}{lll}\mathrm{Or}_{20} & \mathrm{Ab}_{65} & \mathrm{An}_{15} \\
\mathrm{Oa} \\
\mathrm{Or}_{49} & \mathrm{Ab}_{47} & \mathrm{An}_{4}\end{array}$ & $\begin{array}{l}\text { anorthose/sanidine } \\
\text { sodique }\end{array}$ & $\begin{array}{l}\mathrm{Or}_{5} \mathrm{Ab}_{59} \mathrm{An}_{36} \mathrm{a} \\
\mathrm{Or}_{12} \mathrm{Ab}_{68} \mathrm{An}_{20}\end{array}$ & $\begin{array}{l}\text { andésine/oligoclase } \\
\text { potassique }\end{array}$ & \\
\hline $\begin{array}{l}\text { T6 } \\
(16)\end{array}$ & $\begin{array}{lll}\mathrm{Or}_{38} & \mathrm{Ab}_{58} & \mathrm{An}_{4} \mathrm{a} \\
\mathrm{Or}_{50} & \mathrm{Ab}_{47} & \mathrm{An}_{3}\end{array}$ & sanidine sodique & $\mathrm{Or}_{7} \mathrm{Ab}_{72} \mathrm{An}_{21}$ & oligoclase & $\begin{array}{l}\text { sanidine sodique } \\
\text { dominante }\end{array}$ \\
\hline
\end{tabular}

Tab. 2 : Composition chimique et minéralogie des feldspaths dans les téphras de la séquence AL/C3 du maar d'Alleret. Le nombre d'analyses par téphra est indiqué entre parenthèses, p.o. : poids d'oxyde.

Tab. 2: Geochemical and mineralogical compositions of feldspars in the tephras from the AL/C3 sequence of the Alleret maar. Number of analysis per tephra indicated between brackets, p.o.: oxide weight.

lité cyclique, avec des niveaux relativement plus sableux (U4c, U4e) ou moins sableux (U4a/b, U4d, U4f). La courbe des pourcentages de limon présente aussi une allure cyclique, avec des niveaux plus limoneux (U4b, U4d, U4f) ou moins limoneux (U4a, U4c, U4e).

Les valeurs de susceptibilité magnétique sont, dans l'ensemble, relativement élevées dans la partie basse de la séquence entre $-40,50$ et $-36,30 \mathrm{~m}$ (unité $\mathrm{U} 1$ ), avec des valeurs moyennes autour de $400 \times 10^{-8} \mathrm{~m}^{3} \cdot \mathrm{kg}^{-1}$ et de nombreux pics compris entre 600 et $800 \times 10^{-8} \mathrm{~m}^{3} \cdot \mathrm{kg}^{-1}$ (fig. 7). Ces derniers sont liés à la présence de téphras, de sables ponceux remobilisant ces téphras, ou à des apports basaltiques sableux à sablo-graveleux en provenance du bassin versant. Une baisse de la susceptibilité intervient entre $-36,30$ et $-34,40 \mathrm{~m}$ (U2a), passant en moyenne de 400 à $200 \times 10^{-8} \mathrm{~m}^{3} \cdot \mathrm{kg}^{-1}$, avec la présence de pics entre 400 et $700 \times 10^{-8} \mathrm{~m}^{3} \cdot \mathrm{kg}^{-1}$.
Des valeurs de susceptibilité magnétique plus basses et relativement stables aux alentours de $200 \times 10^{-8} \mathrm{~m}^{3} \cdot \mathrm{kg}^{-1}$ sont ensuite comprises entre -34,40 et -33,10 m (U2b), et pourraient correspondre à une réduction des apports particulaires grossiers ferromagnésiens en provenance du bassin versant. De $-33,10$ à $-30,15 \mathrm{~m}$ (unités U2c et U3), les valeurs augmentent et oscillent entre 200 et $500 \times 10^{-8} \mathrm{~m}^{3} \cdot \mathrm{kg}^{-1}$. La courbe montre ensuite de $-30,15$ à $-24,25 \mathrm{~m}$ (unité U4) la présence de trois cycles de hausse relative d'environ $150 \times 10^{-8} \mathrm{~m}^{3} \cdot \mathrm{kg}^{-1}$, les valeurs étant comprises entre 200 et $450 \times 10^{-8} \mathrm{~m}^{3} \cdot \mathrm{kg}^{-1}$, avec des niveaux à susceptibilité magnétique, soit plus forte (U4b, U4d, U4f), soit plus faible (U4a, U4c, U4e).

Dans le cas du maar d'Alleret, compte tenu de son bassin versant silicaté, les valeurs de carbone total sont essentiellement représentatives du carbone organique, les teneurs en carbone inorganique présent dans le carbonate

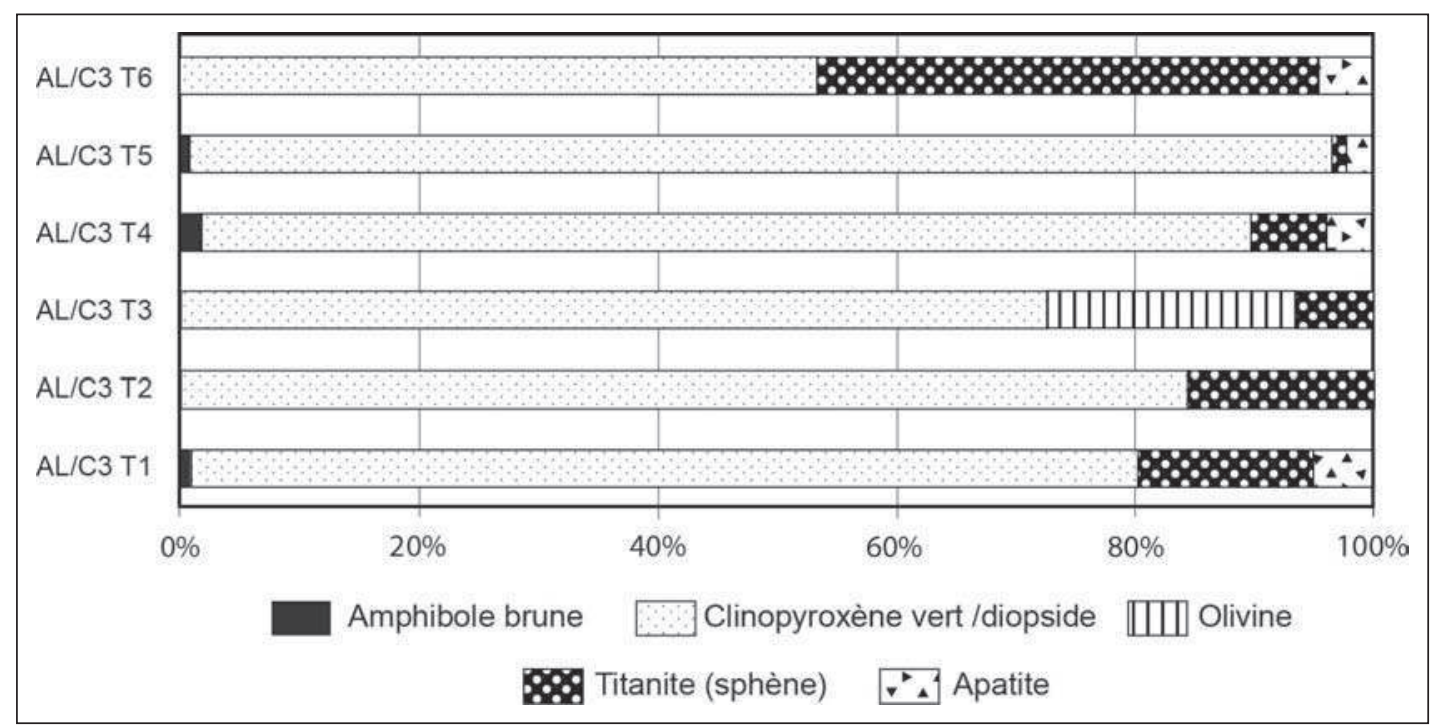

Fig. 6 : Cortège des minéraux lourds des téphras T1 à T6 du carottage AL/C3 du maar d'Alleret.

Fig. 6: Heavy mineral spectrum of the T1 to T6 tephras from the AL/C 3 core from the Alleret maar. 


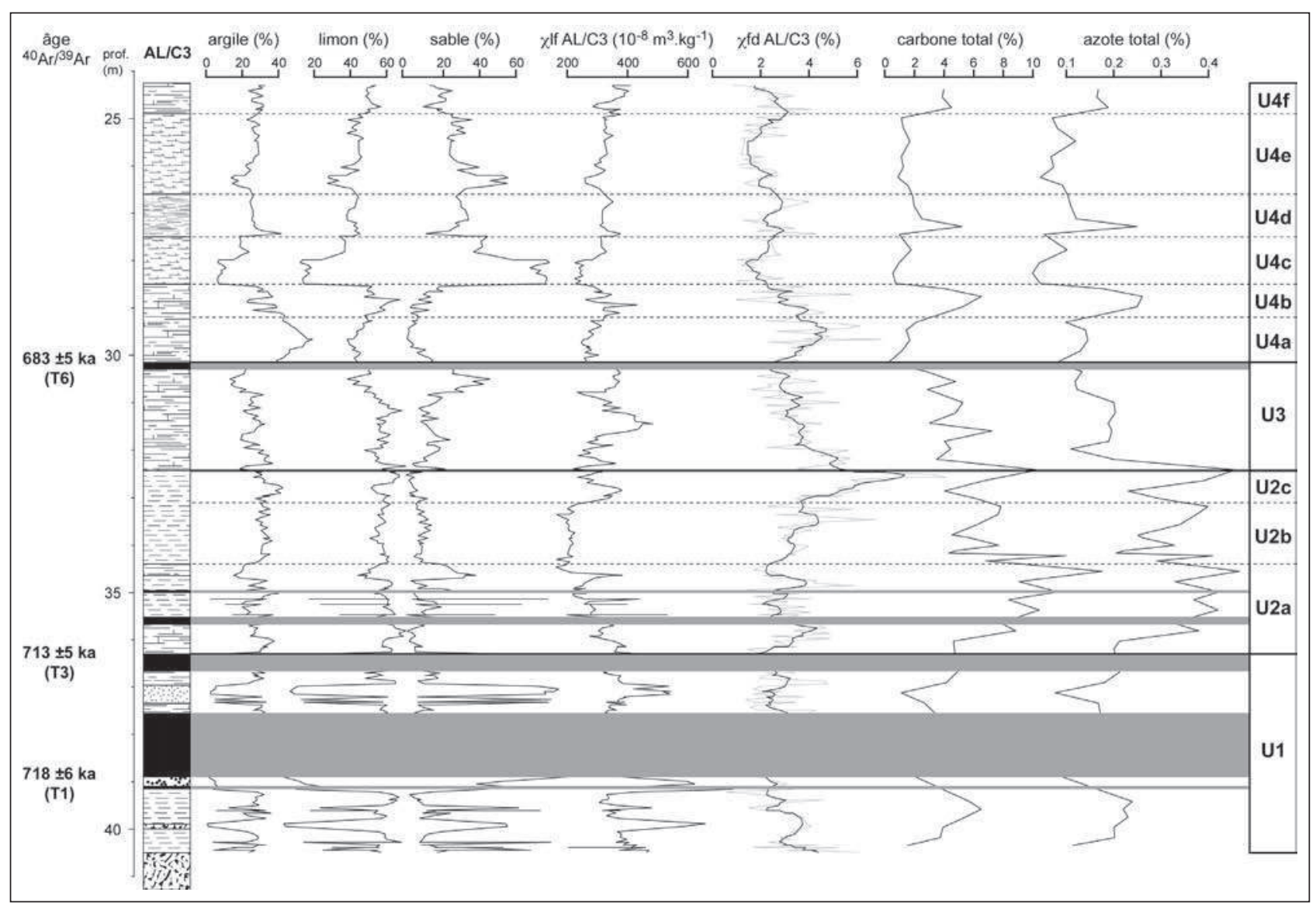

Fig. 7 : Indicateurs sédimentologiques de la séquence sédimentaire AL/C3 du maar d'Alleret.

Fig. 7: Sedimentological proxies of the AL/C3 sedimentary sequence from the Alleret maar.

de calcium étant négligeables. Les courbes de carbone total et d'azote total présentent une évolution relativement similaire (fig. 7).

La base de la séquence entre $-40,50$ et $-36,30 \mathrm{~m}$ (unité U1) montre des valeurs faibles à moyennes de carbone total comprises entre 1 à $6 \%$, et des valeurs d'azote autour de $2 \%$ (fig. 7). Une nette hausse intervient à partir de $-36,30 \mathrm{~m}$, avec des valeurs maximales de carbone et d'azote respectivement comprises entre 10 et $15 \%$ et entre 4 et $5 \%$ de $-36,30$ à $-32,50 \mathrm{~m}$ (unité U2). On observe ensuite une baisse générale des taux de carbone et d'azote respectivement de 8 à $3 \%$ et de 4 à $1 \%$ de $-32,50$ à $-30,15 \mathrm{~m}$ (unité U3). Entre - 30,15 et -24,25 m (unité U4), les valeurs de carbone et d'azote présentent une évolution cyclique, avec la présence de trois cycles montrant des variations d'un ratio de 4 à 5 , passant respectivement de $1 \%$ et $0,5 \%$ (U4a, U4c, U4e) à 4-6\% et 2-2,5\%o (U4b, U4d, U4f).

\section{4 - INTERPRÉTATION DES DONNÉES POLLI- NIQUES}

La base de la séquence sédimentaire du sondage AL/ C3 de - 40,50 à -24,25 m enregistre le développement de systèmes forestiers typiques d'un interglaciaire, auquel succède une période glaciaire caractérisée par la présence d'une végétation de type steppe-toundra (fig. 8).

Le début de la séquence se caractérise par le développement d'une forêt riparienne (Alnus, Myrica, Fraxinus,
Ulmus, Zelkova, Salix) de -40,50 m à -39,50 m (AllC3-1 à AllC3-3). De -36,30 à -32,50 m (AllC3-5 à AllC3$10)$, on constate un optimum climatique avec le développement d'une forêt caducifoliée mixte à Quercus et Carpinus betulus dominants, et, plus en altitude, celui de la forêt à Abies. On note la présence de Zelkova, Carya, Pterocarya et Liquidambar, taxons participant aux forêts tempérées chaudes d'Europe jusqu'au début du Pléistocène moyen. Le développement de -32,50 à -30,15 m (AllC3-11 à AllC3-16) d'une forêt montagnarde à conifères (Pinus, Picea, Abies, Cathaya, Tsuga) signale un changement climatique (baisse des températures). Au cours de cet interglaciaire d'Alleret, on observe trois phases de réduction forestière (AllC3-7, AllC3-12 et AllC3-14). A partir de -30,15 m et jusqu'à - $25 \mathrm{~m}$, une végétation de type steppe/toundra, dominée par les Poaceae, Artemisia, Chenopodiaceae-type et Cupressaceae, est enregistrée. Elle correspond à une phase glaciaire postérieure à $680 \mathrm{ka}$. On y observe trois brefs épisodes de réchauffement (AllC3-18, AllC3-20 et AllC3-22) caractérisés par une faible expansion forestière marquant des changements climatiques rapides.

La composition floristique des systèmes forestiers successifs au cours de l'interglaciaire montre l'importance de Carpinus betulus, la présence de Cathaya, Tsuga, Carya, Liquidambar, Nyssa, Pterocarya, Syringa, Celtis et Zelkova et la grande diversité de la flore herbacée. Ces caractéristiques font la singularité de l'interglaciaire enregistré à Alleret et le distinguent fortement de l'inter- 


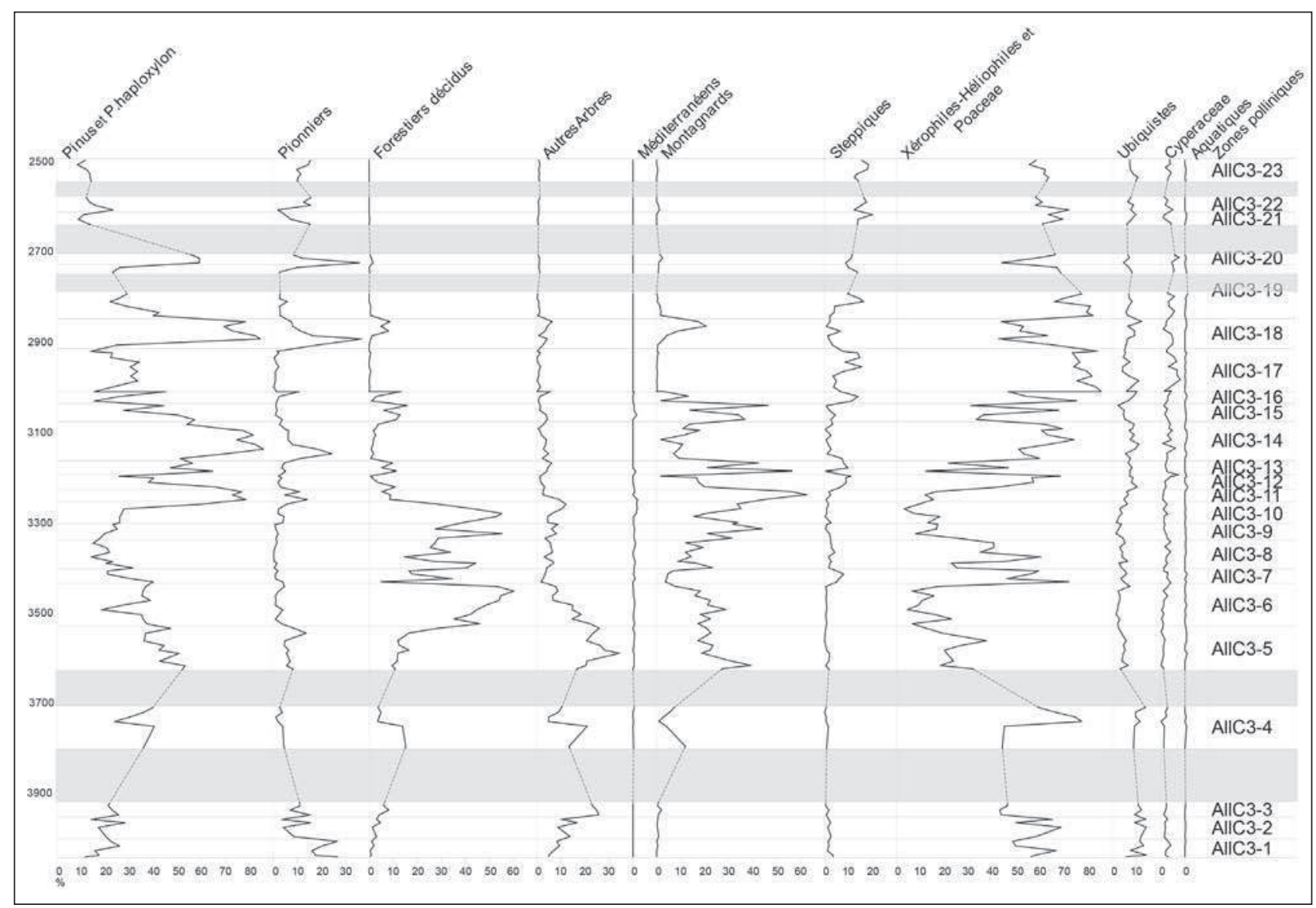

Fig. 8 : Diagramme palynologique simplifié de la partie inférieure (-40,50 à -25 m) du carottage AL/C3 du maar d'Alleret.

Les bandes grisées indiquent les zones sans prélèvement (téphras T2, T3 et zones perturbées par le carottage). Les taxons ont été regroupés selon leur signification écologique : 1/ Pinus et Pinus haploxylon-type, 2/ Arbres pionniers (Cupressaceae, Betula, Hippophae rhamnoides), 3 / Arbres de la forêt caducifoliée (Acer, Ilex, Hedera, Carpinus betulus-type, Carpinus orientalis-type, Buxus, Fagus, Quercus caducifolié-type, Carya, Pterocarya, Viscum, Nyssa, Tilia), 4/ Autres arbres (Taxus, Alnus, Corylus, Sambucus, Viburnum, Liquidambar, Juglans, Myrica, Fraxinus excelsior-type, Populus, Salix, Ribes, Celtis, Ulmus, Zelkova-type), 5/ Taxons méditerranéens (Quercus ilex-type, Ligustrum, Olea, Syringa), 6/ Conifères d'altitude (Abies, Cathaya, Picea, Tsuga), 7/ Herbacées steppiques (Ephedra distachya-type, Ephedra fragilis-type, Artemisia, Chenopodiaceae-type), 8/ oaceae et Herbacées xérophiles-héliophiles (Asteroideae, Anthemis-type, Aster-type, Centaurea, Centaurea montana-type, Centaurea nigra-type, Centaurea scabiosa-type, Cirsium-type, Cichorioideae, Caryophyllaceae, Herniaria, Helianthemum, Erodium, Plantago, Plantago lanceolata-type, Armeria-type), 9/ Autres herbacées (Apiaceae, Boraginaceae, Echium-type, Brassicaceae, Campanulaceae, Calystegia, Convolvulus, Crassulaceae, Knautia, Scabiosa-type, Ericaceae, Euphorbia, Mercurialis, Fabaceae, Gentianaceae, Centaurium-type, Geranium, Lamiaceae 3colpé-type, Lamiaceae 6colpé-type, Liliaceae, Linum, Onagraceae, Limonium-type, Polygonum, Polygonum aviculare-type, Polygonum bistorta-type, Rumex, Primulaceae, Ranunculaceae, Helleborus-type, Thalictrum, Rosaceae, Alchemilla-type, Filipendula, Potentilla-type, Sanguisorba minor, Sanguisorba officinalis, Rubiaceae, Saxifragaceae, Saxifraga aizoon-type, Scrofulariaceae, Euphrasia-type, Solanaceae, Urticaceae, Valerianaceae, Violaceae), 10/ Cyperaceae, 11/ Herbacées de milieux humides (Alisma, Callitriche, Myriophyllum verticillatum-type, Lemna, Lythraceae, Menyanthes, Nuphar, Nymphaea, Rumex aquaticus-type, Potamogeton, Scheuchzeria palustris, Sparganium-Typha-type, Typha latifolia).

Fig. 8: Summary pollen diagram from the lower part $(-40.50$ to $-25 \mathrm{~m})$ of AL/C3 core from the Alleret maar. The greved bands indicate zones without samples (tephras T2, T3 and disturbed zones by drilling). Taxa are grouped according to their ecological significance as follows: 1/ Pinus and Pinus haploxylon-type, 2/ Pioneers trees (Cupressaceae, Betula, Hippophae rhamnoides), 3/ Deciduous forest trees (Acer, Ilex, Hedera, Carpinus betulus-type, Carpinus orientalis-type, Buxus, Fagus, deciduous Quercus, Carya, Pterocarya, Viscum, Nyssa, Tilia), 4/ Other deciduous trees (Taxus, Alnus, Corylus, Sambucus, Viburnum, Liquidambar, Juglans, Myrica, Fraxinus excelsior-type, Populus, Salix, Ribes, Celtis, Ulmus, Zelkova-type), 5/ Mediterranean elements (Quercus ilex-type, Ligustrum, Olea, Syringa), 6/Altitudinal coniferous forest (Abies, Cathaya, Picea, Tsuga), 7/ Steppic herbs (Ephedra distachya-type, Ephedra fragilis-type, Artemisia, Chenopodiaceae-type), 8/ Poaceae and xerophilous-heliophilous herbs (Asteroideae, Anthemis-type, Astertype, Centaurea, Centaurea montana-type, Centaurea nigra-type, Centaurea scabiosa-type, Cirsium-type, Cichorioideae, Caryophyllaceae, Herniaria, Helianthemum, Erodium, Plantago, Plantago lanceolata-type, Armeria-type), 9/ Other herbs (Apiaceae, Boraginaceae, Echium-type, Brassicaceae, Campanulaceae, Calystegia, Convolvulus, Crassulaceae, Knautia, Scabiosa-type, Ericaceae, Euphorbia, Mercurialis, Fabaceae, Gentianaceae, Centaurium-type, Geranium, Lamiaceae 3colpate-type, Lamiaceae 6colpate-type, Liliaceae, Linum, Onagraceae, Limonium-type, Polygonum, Polygonum aviculare-type, Polygonum bistorta-type, Rumex, Primulaceae, Ranunculaceae, Helleborus-type, Thalictrum, Rosaceae, Alchemilla-type, Filipendula, Potentilla-type, Sanguisorba minor, Sanguisorba officinalis, Rubiaceae, Saxifragaceae, Saxifraga aizoon-type, Scrofulariaceae, Euphrasia-type, Solanaceae, Urticaceae, Valerianaceae, Violaceae), 10/ Cyperaceae, 11/ Aquatic plants (Alisma, Callitriche, Myriophyllum verticillatum-type, Lemna, Lythraceae, Menyanthes, Nuphar, Nymphaea, Rumex aquaticus-type, Potamogeton, Scheuchzeria palustris, Sparganium-Typha-type, Typha latifolia).

glaciaire de Praclaux corrélé avec le stade isotopique marin 11 (Reille \& Beaulieu, 1995) et des interglaciaires reconnus dans le Pléistocène inférieur régional (Elhaï, 1969 ; Brun, 1971 ; Ablin, 1991). Cette confrontation des séquences du Massif central souligne l'importance de la séquence d'Alleret pour la compréhension des changements paléoenvironnementaux au cours du début du Pléistocène moyen.

\section{5 - DISCUSSION}

\section{1 - ORIGINE DES TÉPHRAS}

Les téphras du carottage $\mathrm{AL} / \mathrm{C} 3$ sont tous très vraisemblablement attribuables au volcanisme du Sancy (Mont Dore), situé à $60 \mathrm{~km}$ au NO (fig. 1), et qui fut actif entre 900 et 250 ka (Cantagrel \& Baubron, 1983 ; 
Féraud et al., 1990 ; Pastre \& Cantagrel, 2001 ; Nomade et al., 2012). Il s'agit du seul appareil volcanique d'Europe occidentale en activité à cette époque ayant pu émettre sur des distances pluri-décakilométriques les épais téphras retrouvés au maar d'Alleret, et notamment le téphra T2, qui atteint $140 \mathrm{~cm}$ d'épaisseur, et qui marque un épisode majeur dans l'histoire du volcan du Sancy. D'autres téphras plus récents provenant de ce volcan ont déjà été observés au sein des remplissages sédimentaires intracratériques de maars pléistocènes du Velay, comme ceux du lac du Bouchet et de Praclaux, situés plus au sud sur le plateau du Devès (Roger et al., 1999), ou dans la partie supérieure de la séquence sédimentaire lacustre du maar d'Alleret (Pastre et al., 2007).

Le volcan du Sancy, constitué de roches alcalines saturées à sous-saturées, a connu quatre principaux cycles d'activité d'environ 100 ka chacun : le cycle I entre 1101 et $1000 \mathrm{ka}$, le cycle II (Super-Besse) entre 818 et $685 \mathrm{ka}$, le cycle III (Neschers) entre 642 et $537 \mathrm{ka}$, et le cycle IV entre 392 et $280 \mathrm{ka}$ (Nomade et al., 2012). Les âges ${ }^{40} \mathrm{Ar} /{ }^{39} \mathrm{Ar}$ obtenus pour les téphras de la base de la séquence sédimentaire d'Alleret permettent de les attribuer à la partie terminale du cycle de Super-Besse. La comparaison de la composition chimique des verres volcaniques des téphras du maar d'Alleret avec celle des verres volcaniques de dépôts pyroclastiques liés à l'activité volcanique pléistocène du Sancy (J.-F. Pastre, données inédites) permet de proposer des corrélations stratigraphiques avec de potentiels volcans sources (fig. 9).

La phase terminale du cycle I est représentée par le dépôt pyroclastique à verre rhyolitique du Bois de la Tour daté à $1010 \pm 2 \mathrm{ka}$ (Nomade et al., 2012). Les premières éruptions du cycle II sont représentées par la séquence du ruisseau de Chareire dont la base et le sommet ont été respectivement datés à $817 \pm 3$ et $736 \pm 4$ ka (Nomade et al., 2012). La mésostase vitreuse des dépôts associés à cette séquence présente une composition trachytique légèrement plus acide que le couple de téphra T2/T3, dont les verres montrent une grande similarité géochimique avec ceux de la coulée pyroclastique ponceuse de la Biche d'après le diagramme silice-alcalins (fig. 9). La datation de cet événement éruptif à $719 \pm 6$ ka s'accorde bien avec l'âge du téphra T3 à $713 \pm 2 \mathrm{ka}$. Toutefois, la composition en minéraux lourds du dépôt de la Biche montre une association clinopyroxène vert $(75 \%) /$ titanite $(20 \%)$, avec comme minéraux accessoires des apatites et des amphiboles brunes (Pastre \& Cantagrel, 2001), plus en adéquation avec la composition minéralogique du téphra T1 (fig. 6). L'âge de ce téphra à $718 \pm 6$ ka est par ailleurs plus proche de celui du dépôt pyroclastique de la Biche.

Les téphras $\mathrm{T} 4$ et $\mathrm{T} 5$, compris entre $683 \pm 5$ et $718 \pm 6 \mathrm{ka}$, correspondraient à des épisodes éruptifs moins différenciés dans la partie terminale du cycle de Super-Besse.

\section{2 - ÉVOLUTION PALÉOENVIRONNEMENTALE}

L'analyse multi-indicateurs des différents signaux sédimentologiques, géochimiques et palynologiques permet de reconstituer l'évolution paléoenvironnementale du maar d'Alleret au début du Pléistocène moyen. Quatre grandes unités stratigraphiques ont été distinguées dans la partie inférieure de la séquence sédimentaire $\mathrm{AL} / \mathrm{C} 3$ (fig. 7).

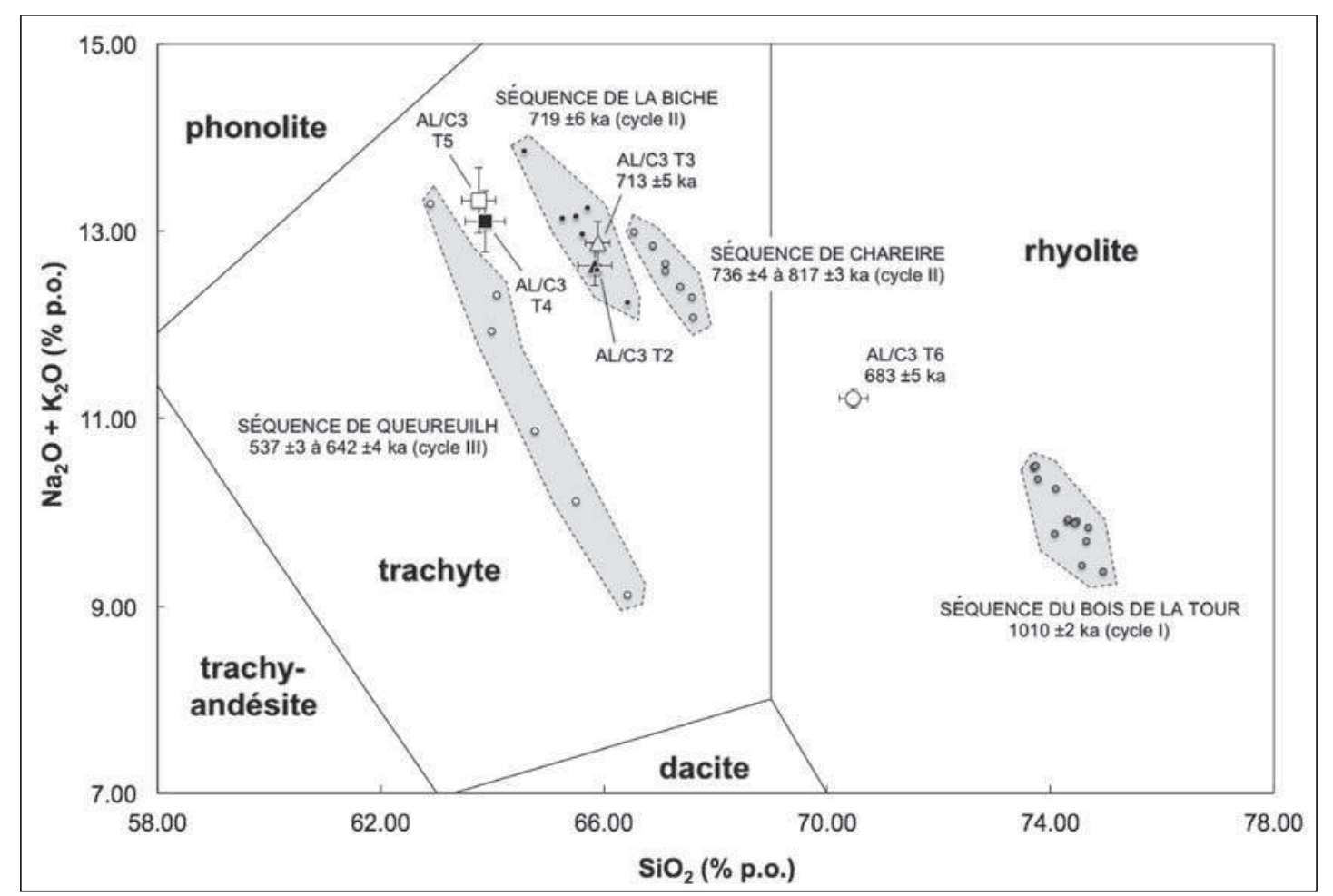

Fig. 9 : Diagramme silice-alcalins des verres volcaniques dans les téphras T2 à T6 du carottage AL/C3 du maar d'Alleret et dans les dépôts pyroclastiques du Sancy avec leurs âges ${ }^{40} \mathrm{Ar} /{ }^{39} \mathrm{Ar}$.

Fig. 9: Total Alkali-Silica diagram of the volcanic glass in the T2 to T6 tephras from the AL/C3 core of the Alleret maar and in the pyroclastic deposits from the Sancy volcano with ${ }^{40} \mathrm{Ar}{ }^{39} \mathrm{Ar}$ dating. 
L'unité 1 (U1), de - 40,5 à -36,3 m de profondeur, correspond au début d'un interglaciaire, caractérisé par une phase de réchauffement climatique et l'érosion post-éruptive des dépôts phréatomagmatiques affleurant autour du cratère du maar, ainsi que par le dépôt de plusieurs téphras d'épaisseur pluri-décimétrique provenant du volcan du Sancy. Les sédiments lacustres sont généralement fins avec de brèves passées sableuses voire graveleuses. Les valeurs plus élevées de susceptibilité magnétique correspondent, soit à ces apports sédimentaires grossiers riches en clastes basaltiques millimétriques à centimétriques, soit à l'émission de téphras à verre de composition trachytique des phases éruptives du Sancy. Les niveaux sédimentaires grossiers correspondent vraisemblablement à des apports détritiques en provenance du bassin versant, et plus particulièrement de l'érosion de l'anneau de tufs basaltiques autour du cratère. Cette morphogenèse post-éruptive a pu être accentuée au début de l'interglaciaire d'Alleret, pendant lequel s'est développée une forêt riparienne où les espèces non arborées peuvent représenter plus de $50 \%$ du cortège pollinique (fig. 8). La couverture végétale encore peu densifiée aurait ainsi pu protéger moins efficacement le substrat de l'érosion mécanique, comme ceci a pu être observé dans le bassin versant du maar du lac du Bouchet, plus au sud sur le plateau du Devès, lors du dernier cycle climatique et du post-glaciaire holocène (Degeai \& Pastre, 2009).

L'unité 2, comprise entre - 36,3 et - 32,5 m de profondeur, correspond à une période de climat tempéré ayant permis le développement d'une forêt à conifères et caducifoliés. Cette unité se caractérise par une sédimentation essentiellement argilo-limoneuse avec quelques passées sableuses en début de période (U2a), indiquant un ralentissement de l'érosion mécanique dans le bassin versant probablement dû à une stabilisation progressive du substrat et des sols par une couverture végétale constituée de forêts décidues. Les valeurs de susceptibilité magnétique décroissent (U2a) puis deviennent relativement basses et stables (U2b), tandis que la hausse du coefficient de susceptibilité magnétique dépendante à la fréquence montre une augmentation du taux de particules superparamagnétiques dans les sédiments, avec un maxima en fin de période (U2c). Ceci traduit probablement un approfondissement des sols et une accentuation de la pédogenèse et des processus d'altération biochimique (Dearing et al., 1996a, 1996b ; Sartori et al., 2005), corrélés à une phase d'organogenèse plus intense comme le montrent les taux de carbone et d'azote dans l'ensemble plus élevés.

L'unité 3, comprise entre - 32,5 et -30,15 m, correspond à la fin d'un interglaciaire, marqué par un refroidissement climatique et une augmentation de la sédimentation détritique qui se traduit par une croissance des taux de sable, accompagnée par une hausse de la susceptibilité magnétique. La décroissance du coefficient de dépendance à la fréquence de la susceptibilité magnétique montre probablement un ralentissement des processus pédogénétiques (Dearing et al., 1996a, 1996b). Cette unité est aussi marquée par une diminution de la production de matière organique et le remplacement de la forêt caducifoliée mixte par une forêt montagnarde.
De - 30,30 à - 18,85 m, l'unité 4 correspond à une période globalement froide durant laquelle s'est développée une végétation steppique. Dans le détail, on peut toutefois observer une variabilité climatique avec une succession de stade et d'interstade. Cette variabilité s'observe dans la granulométrie des sédiments lacustres qui montrent une fluctuation des taux d'argiles, de limons et de sables, avec toutefois des niveaux globalement plus sableux que dans les unités précédentes. Par ailleurs, la variabilité climatique est révélée par des taux de carbone et d'azote qui s'organisent en trois cycles stades/interstades, bien corrélés aux données palynologiques disponibles. Cellesci montrent en effet une alternance entre une végétation de steppe-toundra et de brèves phases d'expansion forestière. De plus, on observe une croissance générale des valeurs de susceptibilité magnétique qui pourrait traduire une plus forte érosion mécanique des matériaux volcaniques superficiels (scories, dépôts phréatomagmatiques) dans le bassin versant, due à une moins bonne protection du substrat par un couvert végétal plus ouvert et des sols moins épais (lithosols) ou érodés.

\section{3 - COMPARAISON INTER-SÉQUENTIELLE}

Les principales unités sédimentaires mises en évidence sur la séquence d'Alleret ont été comparées avec d'autres référentiels glaciels, marins ou continentaux (fig. 10) : les courbes des variations du deutérium, de la concentration en poussières, du dioxyde de carbone $\left(\mathrm{CO}_{2}\right)$ et du méthane $\left(\mathrm{CH}_{4}\right)$ dans les glaces de l'Antarctique à partir des sondages EPICA Dome C (Jouzel et al., 2007 ; Lambert et al., 2008), les teneurs en carbonate de calcium dans les sédiments du nord-est de l'Atlantique à partir du carottage du site ODP 982 (Venz et al., 1999), les variations isotopiques de l'oxygène dans les sédiments marins $\left(\delta^{18} \mathrm{O}\right.$ Benthic Stack LR04, Lisiecki \& Raymo, 2005, 2007), les variations des pourcentages de silice biogénique dans les sédiments lacustres du lac Baïkal d'après les carottages BDP-96-1 et BDP-96-2 (Prokopenko et al., 2006), ainsi que la courbe du taux de pollens arborés de la séquence de Tenaghi Philippon en Grèce (Tzedakis et al., 2006).

Parmi tous ces référentiels, les courbes du lac Baïkal et de EPICA Dome $\mathrm{C}$ présentent la meilleure résolution temporelle, avec un nombre respectif de 17 et 7 points mesurés entre les bornes chronologiques 713 et $718 \mathrm{ka}$, d'une part, et de 68 et 75 points entre 683 et $713 \mathrm{ka}$, d'autre part. Ceci représente respectivement un pas de mesure moyen de 0,3 et $0,71 \mathrm{ka}$ pour la période $718-713 \mathrm{ka}$, et 0,44 et $0,4 \mathrm{ka}$ pour la période $713-683 \mathrm{ka}$. A titre indicatif, sur les mêmes périodes, la courbe granulométrique du pourcentage de sable contenu dans les sédiments intracratériques du maar d'Alleret, utilisée pour les comparaisons avec les autres référentiels paléobioclimatiques, bénéficie respectivement de 25 et 127 points de mesure, soit un pas chronologique moyen de 0,2 et 0,236 ka.

Cette haute résolution temporelle des données obtenues pour la séquence d'Alleret permet d'envisager une analyse comparative relativement fine avec les autres marqueurs. Toutefois, les âges radio-isotopiques ${ }^{40} \mathrm{Ar} /{ }^{39} \mathrm{Ar}$ ne peuvent pas être directement comparés aux 


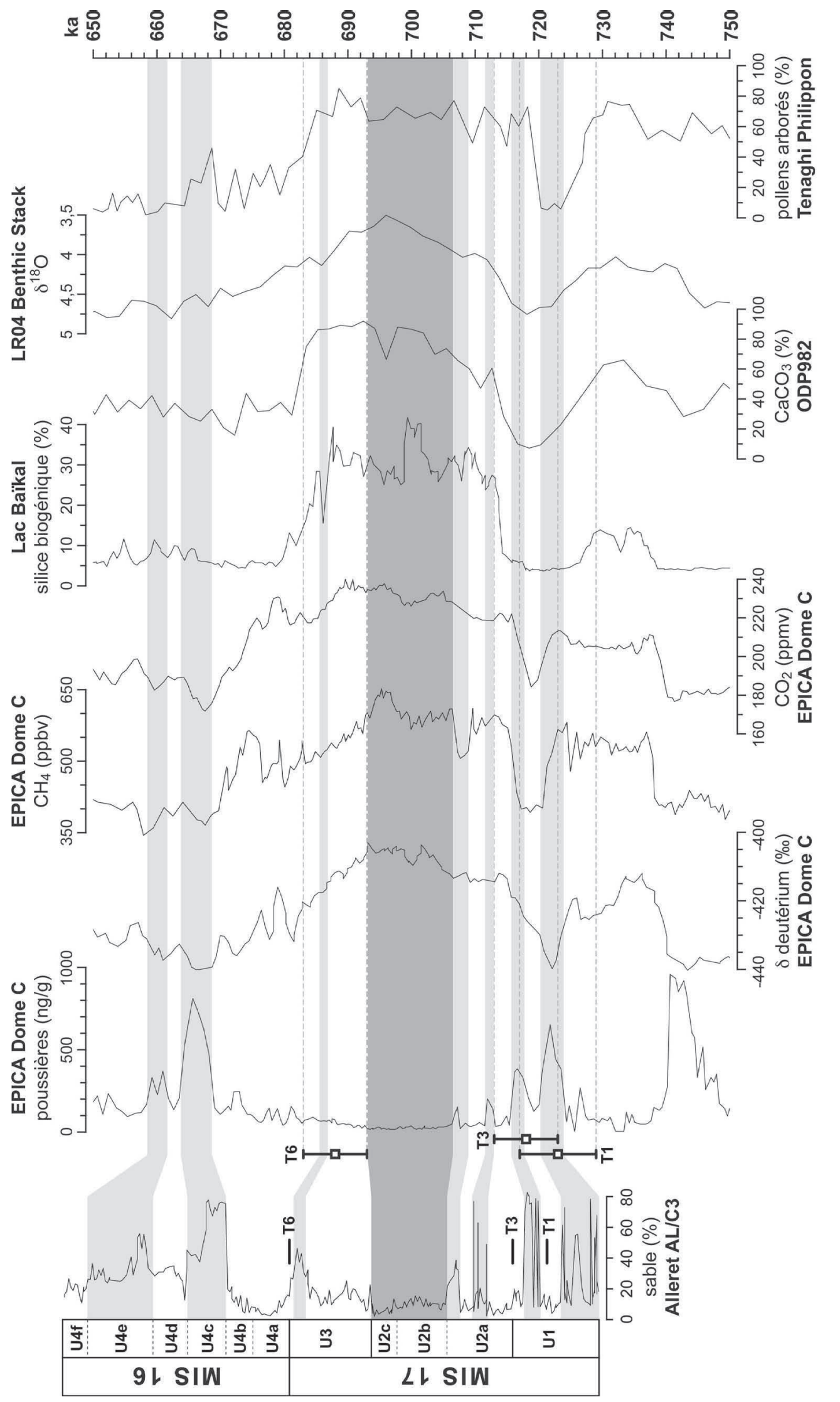

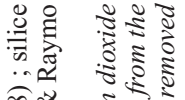

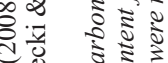

3.

해을

든

ใิ

वृ.

E.

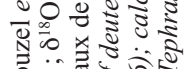

政娄

क्षे

บ을

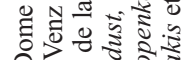

a

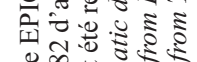

نㅎํ

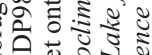

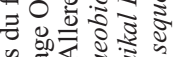

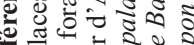
4 ธै

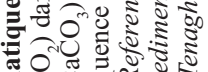
U U U

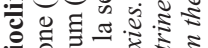

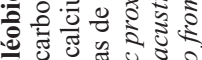

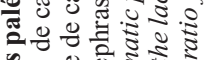
屯ैं 要 \%

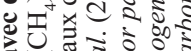
产泀

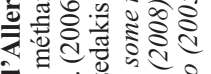

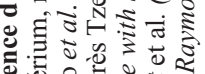

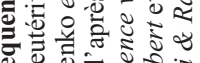
क्षण एँ

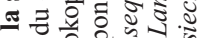
ㄴ.

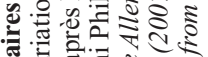

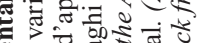
为可 ठ。

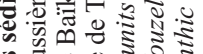

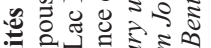

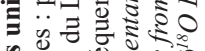

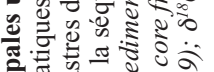
을.

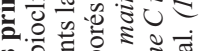

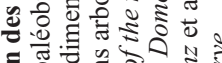

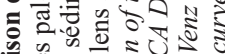

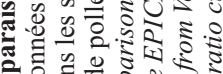

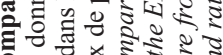

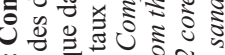


âges obtenus par des méthodes de calibration astronomique, comme celles utilisées pour les référentiels paléoclimatiques des glaces polaires et des sédiments marins (Renne et al., 1998 ; Min et al., 2000 ; Nomade et al., 2010). Ainsi, afin de faciliter les comparaisons avec la chronologie des autres référentiels, les âges moyens des téphras T1, T3 et T6 ont été recalculés avec un âge de 1,201 Ma pour le standard ACs-2 (Nomade et al., 2005 ; Kuiper et al., 2008) et selon la constante de désintégration totale du potassium suggérée par Kwon et al. (2002). Ceci aboutit à un vieillissement d'environ $0,7 \%$ pour tous les âges moyens. Les limites d'âge reportées sur la figure 10 tiennent comptent de ces corrections.

La période charnière entre la fin du glaciaire SIM 18 et le début de l'interglaciaire SIM 17 montre la présence de quatre pics décroissants de concentration en poussières dans les glaces du forage EPICA Dome C. En tenant compte des âges des téphras T1 et T3, les niveaux à sables grossiers observés dans les unités U1 et U2a de la séquence d'Alleret ont été rattachés à ces pics de poussières. Ces niveaux sableux pourraient par ailleurs montrer que les quatre brèves péjorations climatiques reconnues dans les glaces de l'Antarctique à la transition entre les stades isotopiques SIM 18/17 aux alentours de 724-720, 718-716, 711, et 708-706 ka ont eu un impact mondial et ont été ressenties en Europe occidentale.

L'interglaciaire du stade isotopique marin 17 est bien enregistré par tous les référentiels paléobioclimatiques et se traduit par des taux élevés de pollens arborés à Tenaghi Philippon, un accroissement de la production de silice biogénique au lac Baïkal, de très faibles taux de poussières déposées sur l'inlandsis antarctique (EPICA Dome C), et, plus généralement, par une hausse globale des températures d'après les données géochimiques dans les sédiments marins $\left(\delta^{18} \mathrm{O}\right.$ du Benthic Stack LR04 et $\mathrm{CaCO}_{3}$ du forage ODP982) et dans les glaces polaires ( $\delta$-deutérium, $\mathrm{CH}_{4}$ et $\mathrm{CO}_{2}$ du forage EPICA Dome C). Au maar d'Alleret, l'organisation de l'interglaciaire SIM 17 dans la partie inférieure de la séquence se caractérise par la présence d'un optimum climatique (unités U2b/c). Celui-ci se retrouve sur la plupart des autres référentiels entre 693 et $706 \mathrm{ka}$, avec, d'une part, des valeurs maximales de $\delta$-deutérium et de concentration en méthane et des concentrations minimales en poussières dans les glaces du pôle sud (EPICA Dome C), et, d'autre part, des valeurs minimales de $\delta^{18} \mathrm{O}$ (Benthic Stack LR04). Par ailleurs, cet optimum englobe le niveau élevé de silice biogénique observé dans les sédiments du lac Baïkal entre 698 et 702 ka.

Les référentiels paléobioclimatiques montrent ensuite une baisse globale progressive des températures. Cette phase de refroidissement climatique d'une dizaine de millénaires, qui s'amorce, selon les proxies, aux alentours de 690-695 ka, se retrouve au maar d'Alleret au niveau de l'unité U3, durant laquelle se développe une forêt montagnarde à conifère. Dans la partie terminale de cette unité, peu avant la transition avec la période glaciaire du SIM 16, on observe un pic de sable qui a été chronologiquement rattaché, à titre d'hypothèse, à la brève et brusque baisse de production de silice biogénique dans le lac Baïkal vers $686 \mathrm{ka}$.
Les sédiments de l'unité U4 montrent dans l'ensemble une granulométrie plus grossière, qui traduit vraisemblablement une activité morphodynamique détritique plus marquée dans le bassin versant typique d'une phase de rhéxistasie, dans un contexte climatique général de période froide, correspondant au glaciaire SIM 16 d'après les données paléobioclimatiques. Durant les périodes glaciaires du Quaternaire, les hauts plateaux du Massif central ont évolué dans un système morphogénique périglaciaire (Valadas, 1983). Au sein de cette unité U4, la sous-unité U4c, caractérisée par un fort épisode détritique, a été rattachée au pic important de poussières piégées dans les glaces antarctiques du Dome $\mathrm{C}$ entre 664 et $669 \mathrm{ka}$. Un deuxième épisode de détritisme moins marqué que le précédent (unité U4e) est rattaché au double pic de poussières glaciaires situé entre 658 et $662 \mathrm{ka}$. Les sédiments moins grossiers des sous-unités $\mathrm{U} 4 \mathrm{a} / \mathrm{b}, \mathrm{U} 4 \mathrm{~d}$ et $\mathrm{U} 4 \mathrm{f}$ se sont probablement déposés pendant des périodes interstadiaires, caractérisées par une dynamique éolienne moins intense et des températures moins froides au-dessus de l'inlandsis antarctique, comme semblent respectivement en témoigner les valeurs généralement plus basses de concentration en poussières et les teneurs relativement plus élevées en deutérium, méthane et $\mathrm{CO}_{2}$ enregistrées par les glaces du forage EPICA Dome $\mathrm{C}$.

\section{7 - CONCLUSION}

Le maar d'Alleret contient l'une des premières séquences sédimentaires lacustres reconnues en milieu continental en Europe de l'ouest pour la période du début du Pléistocène moyen. La présence de plusieurs niveaux de retombées de cendres volcaniques dans le carottage $\mathrm{AL} / \mathrm{C} 3$ permet d'établir une téphrochronologie par datations ${ }^{40} \mathrm{Ar} /{ }^{39} \mathrm{Ar}$ laser de trois téphras issus des éruptions du massif du Sancy (cycle II Super-Besse), confirmant l'intérêt majeur de ce volcan pour dater les événements pléistocènes du Massif central, et, plus généralement, d'Europe occidentale. La datation de ces téphras fait du maar d'Alleret la séquence sédimentaire continentale la mieux datée en Europe pour le début du Pléistocène moyen.

Les données chronostratigraphiques et palynologiques obtenues pour la partie inférieure de la séquence sédimentaire lacustre du maar d'Alleret mettent en évidence au début de la seconde phase de la Mid-Pleistocene Transition un interglaciaire d'une durée d'environ $40 \mathrm{ka}$ attribué au stade SIM 17, ainsi que l'existence d'une transition interglaciaire-glaciaire vers $683 \pm 5$ ka pour les stades isotopiques marins 17/16. Par ailleurs, les analyses sédimentologiques et géochimiques permettent de mieux comprendre l'évolution paléoenvironnementale post-éruptive du maar, et notamment les épisodes d'alimentation détritique sableuse au sein du remplissage sédimentaire intracratérique dus à une hausse de l'érosion mécanique dans le bassin versant, ainsi que le développement de phases de biostasie durant lesquelles sont favorisées l'altération chimique et la pédogenèse. 
L'application de la démarche multi-analytique développée dans ce travail au sondage $\mathrm{AL} / \mathrm{C} 4$ permettra, à l'avenir, de compléter l'étude paléoenvironnementale et chronostratigraphique de la séquence du maar d'Alleret. Ces nouvelles données devraient notamment permettre, d'une part, de compléter l'analyse de la partie supérieure de la période glaciaire du stade SIM 16, dont la variabilité cyclique en stade/interstade a été observée dans le carottage $\mathrm{AL} / \mathrm{C} 3$, et, d'autre part, de renseigner la transition entre les stades MIS 16 et 15 , ce dernier ayant déjà été mis en évidence au maar d'Alleret par Pastre et al. (2007) dans les quinze mètres sommitaux du remplissage sédimentaire intracratérique.

Compte tenu de la haute résolution temporelle des marqueurs sédimentologiques utilisés dans ce travail et de leur très bonne adéquation avec les différents référentiels paléobioclimatiques disponibles dans des secteurs géographiquement variés pour la période étudiée, la séquence sédimentaire lacustre du maar d'Alleret pourrait servir de référence majeure à l'échelle mondiale pour cette période-clé qu'est le début du Pléistocène moyen dans l'histoire du Quaternaire.

\section{REMERCIEMENTS}

Les auteurs tiennent à remercier le BRGM, le CEA et le CNRS, sans lesquels ce travail n'aurait pu être réalisé. Nous remercions particulièrement Daniel Dupuy, ancien chef-sondeur du BRGM qui a pu permettre de monter techniquement le programme de carottage sur le maar d'Alleret et d'autres maars. Nos remerciements sincères vont aussi à l'égard de Ph. Rossi (Carte géologique de la France) qui a permis la réalisation de ces sondages. Nos remerciements chaleureux vont également à tous ceux qui ont contribué, par leur soutien, leur aide ou leur encouragement, à la réalisation de ce travail : G. Beaufort (commune de Saint-Privat-du-Dragon), J.-M. Cantagrel, J.-J. Delannoy, la famille Echobart, E. Egly, F. Couffignal, M. Fialin, G. Guizoarn, Ch. Le Cour, J.-F. Lozach, la famille Perret, E. Poux, F. Quesnel, Ph. Rocher, P. Soulis, Y. Vernhet. Nous remercions enfin les deux relecteurs anonymes pour leurs remarques constructives et pertinentes.

\section{RÉFÉRENCES BIBLIOGRAPHIQUES}

ABLIN D., 1991 - Analyse pollinique des dépôts lacustres de Ceyssac, Plio-Pléistocène du Velay (Massif central, France). Cahiers de Micropaléontologie, 6 (1), 21-38.

ANTOINE P., LIMONDIN-LOZOUET N., CHAUSSÉ C., LAUTRIDOU J.-P., PASTRE J.-F., AUGUSTE P., BAHAIN J.-J., FALGUÈRES C. \& GALEHB B., 2007 - Pleistocene fluvial terraces from northern France (Seine, Yonne, Somme): synthesis and new results. Quaternary Science Reviews, 26 (22-24), 2701-2723.

BERGER A. \& LOUTRE M.-F., 2010 - Modeling the 100-kyr glacialinterglacial cycles. Global and Planetary Change, 72 (4), 275-281.

BRIDGLAND D. \& WESTAWAY R., 2008 - Climatically controlled river terrace staircases: a worldwide Quaternary phenomenon. Geomorphology, 98 (3-4), 285-315.

BRUN A., 1971 - Les applications de la palynologie à l'étude géodynamique du massif volcanique du Mont-Dore. Thèse de Doctorat d'Etat, Université Paris 6 Pierre et Marie Curie, Paris, 244 p.
CANTAGREL J.-M. \& BAUBRON J.-C., 1983 - Chronologie K-Ar des éruptions dans le massif volcanique des Mont-Dore : implications volcanologiques. Géologie de la France, 2 (1), 123-142.

CLARK P.U., ARCHER D., POLLARD D., BLUM J.D. RIAL J.A., BROVKIN V., MIX A.C., PISIAS N.G. \& ROY M., 2006 - The middle Pleistocene transition: characteristics, mechanisms, and implications for long-term changes in atmospheric $\mathrm{pCO}_{2}$. Quaternary Science Reviews, 25 (23-24), 3150-3184.

DE BEAULIEU J.-L. \& REILLE M., 1992 - Long Pleistocene pollen sequences from the Velay Plateau (Massif Central, France). I. Ribains maar. Vegetation History and Archaeobotany, 1 (4), 233-242.

DE BEAULIEU J.-L., ANDRIEU-PONEL V., REILLE M., GRÜGER E., TZEDAKIS C. \& SVOBODOVA H., 2001 - An attempt at correlation between the Velay pollen sequence and the Middle Pleistocene stratigraphy from central Europe. Quaternary Science Reviews, 20 (16-17), 1593-1602.

DEARING J.A., DANN R.J.L., HAY K., LEES J.A., LOVELAND P.J., MAHER B.A. \& O'GRADY K., 1996a - Frequency-dependent susceptibility measurements of environmental materials. Geophysical Journal International, 124 (1), 228-240.

DEARING J.A., HAY K.L., BABAN S.M.J., HUDDLESTON A.S., WELLINGTON E.M.H. \& LOVELAND P.J., 1996b - Magnetic susceptibility of soil: an evaluation of conflicting theories using a national data set. Geophysical Journal International, 127 (3), 728-734.

DEGEAI J.-P., 2004a - Mesure de l'érosion post-éruptive autour des cratères de maars en inversion de relief dans le Massif central français. Géomorphologie : Relief, Processus, Environnement, 10 (4), 285-304.

DEGEAI J.-P., 2004b - Les lacs de cratère volcanique : un exemple dans le Massif central français. In D. Mercier (ed.), Le commentaire de paysages en géographie physique. Armand Colin, Paris, 84-87.

DEGEAI J.-P. \& PASTRE J.-F., 2009 - Impacts environnementaux sur l'érosion des sols au Pléistocène supérieur et à l'Holocène dans le cratère de maar du Lac du Bouchet (Massif central, France). Quaternaire, 20 (2), 149-159.

DENNELL R.W., 2003 - Dispersal and colonisation, long and short chronologies: how continuous is the Early Pleistocene record for hominids outside East Africa? Journal of Human Evolution, 45 (6), 421-440.

DODONOV A.E., 2005 - The stratigraphic transition and suggested boundary between the Early and Middle Pleistocene in the loess record of northern Eurasia. In M.J. Head \& P.L. Gibbard (eds.), Early-Middle Pleistocene transitions: the land-ocean evidence. Special Publication - Geological Society of London, 247. The Geological Society, London \& AAPG Bookstore, Tulsa, 209-219.

ELHAÏ H., 1969 - La flore sporo-pollinique du gisement villafranchien de Senèze (Massif central, France). Pollen et Spores, 11 (1), 127-139.

FAEGRI K. \& IVERSEN J., 1989 - Textbook of pollen analysis. $4^{\text {th }}$ edition. John Wiley and Sons, Chichester, $328 \mathrm{p}$.

FÉRAUD G., LO BELLO P., HALL C.M., CANTRAGREL J.-M., YORK D. \& BERNAT M., 1990 - Direct dating of Plio-Quaternary pumices by ${ }^{40} \mathrm{Ar} /{ }^{39} \mathrm{Ar}$ step-heating and single-grain laser fusion methods: the example of the Monts-Dore massif (Massif Central, France). Journal of Volcanology and Geothermal Research, 40 (1), 39-53.

FOURIS M., 1989 - Les sites villafranchiens du Devès et les basaltes de la vallée de l'Allier : application de la méthode K/Ar. Thèse de Doctorat, Université Blaise Pascal, Clermont-Ferrand, 210 p.

GIBBARD P.L. \& LEWIN J., 2009 - River incision and terrace formation in the late Cenozoic of Europe. Tectonophysics, 474 (1-2), 41-55.

GUÉRIN B., 1993 - Pollen et allergies. Allerbio, Varennes-en-Argonne, $279 \mathrm{p}$.

HEAD M.J. \& GIBBARD P.L., 2005 - Early-Middle Pleistocene transitions: an overview and recommandation for the defining boundary. In M.J. Head \& P.L. Gibbard (eds.), Early-Middle Pleistocene Transitions: The Land-Ocean Evidence. Special Publication - Geological Society of London, 247. The Geological Society, London \& AAPG Bookstore, Tulsa, 1-18.

HUNTLEY B. \& BIRKS H.J.B., 1983 - An atlas of past and present pollen maps for Europe: 0-13000 years ago. Cambridge University Press, Cambridge, $667 \mathrm{p}$.

JOUZEL J., MASSON-DELMOTTE V., CATTANI G., DREYFUS G., FALOURD S., HOFFMAN G., MINSTER B., NOUET J., BARNOLA J.-M., CHAPPELLAZ J., FISCHER H., GALLET J.-C., JOHNESEN S., LEUENBERGER M., LOULERGUE L., LUETHI D., OERTER H., PARRENIN F., RAISBECK G., RAYNUAD D., SCHILT A., SCHWANDER J., SELMO E., SOUCHEZ R., SPAHNI R., STAUFFER B., STEFFENSEN J.P., STENNI B., STOCKER T.F., TISON J.L., WERNER M. \& WOLFF E.W., 2007 - Orbital and millennial Antarctic climate variability over the Past 800,000 years. Science, 317 (5839), 793-797. 
KUIPER K.F., DEINO A., HILGEN F.J., KRIJGSMAN W., RENNE P.R. \& WIJBRANS J.R., 2008 - Synchronizing rock clocks of earth history. Science, 320 (5875), 500-504.

KWON J., MIN K., BICKEL P. \& RENNE P.R., 2002 - Statistical methods for jointly estimating decay the constant of ${ }^{40} \mathrm{~K}$ and age of a dating standard. Mathematical Geology, 34 (4), 457-474.

LAMBERT F., DELMONTE B., PETIT J.-R., BIGLER M., KAUFMAN P.R., HUTTERLI M.A. STOCKER T.F., RUTH U., STEFFENSEN J.P. \& MAGGI V., 2008 - Dust-climate couplings over the past 800,000 years from the EPICA Dome C ice core. Nature, 452 (7187), 616-619.

LISIECKI L.E. \& RAYMO M.E., 2005 - A Pliocene-Pleistocene stack of 57 globally distributed benthic $\delta^{18} \mathrm{O}$ records. Paleoceanography, 20 (1), 1-17.

LISIECKI L.E. \& RAYMO M.E., 2007 - Plio-Pleistocene climate evolution: trends and transitions in glacial cycle dynamics. Quaternary Science Reviews, 26 (1-2), 56-69.

LORENZ V., 1973 - On the formation of maars. Bulletin Volcanologique, 37 (2), 183-204

LORENZ V., 1986 - On the growth of maars and diatremes and its relevance to the formation of tuff rings. Bulletin of Volcanology, 48 (1), 265-274.

LOURENS L.J., HILGEN F.J., LASKAR J., SHACKLETON N.J. \& WILSON D., 2004 - The Neogene Period. In F.M. Gradstein J.G. Ogg \& A.G. Smith (eds.), A Geologic Time Scale. Cambridge University Press, Cambridge, 409-440.

MAIORANO P. \& MARINO M., 2004 - Calcareous nannofossil bioevents and environmental control on temporal and spatial patterns a the early-middle Pleistocene. Marine Micropaleontology, 53 (3-4), 405-422.

MASLIN M.A. \& RIDGWELL A.J., 2005 - Mid-Pleistocene Revolution and the "eccentricity myth". In M.J. Head \& P.L. Gibbard (eds.), Early-Middle Pleistocene Transitions: The Land-Ocean Evidence. Special Publication - Geological Society of London, 247. The Geological Society, London \& AAPG Bookstore, Tulsa, 19-34.

MIN K., MUNDIL R., RENNE P.R. \& LUDWIG K.R., 2000 - A test for systematic errors in ${ }^{40} \mathrm{Ar} /{ }^{39} \mathrm{Ar}$ geochronology through comparison with $\mathrm{U}-\mathrm{Pb}$ analysis of a $1.1 \mathrm{Ga}$ rhyolite. Geochimica et Cosmochimica Acta, 64 (1), 73-98.

MUDELSEE M. \& STATTEGGER K., 1997 - Exploring the structure of the mid-Pleistocene revolution with advance methods of timeseries analysis. Geologische Rundschau, 86 (2), 499-511.

NOMADE S., RENNE P.R., VOGEL N., DEINO A.L., SHARP W.D., BECKER T.A., JAOUNI A. R. \& MUNDIL R. 2005 - Alder Creek sanidine (ACs-2): A Quaternary ${ }^{40} \mathrm{Ar} /{ }^{39} \mathrm{Ar}$ dating standard tied to the Cobb Mountain geomagnetic event. Chemical Geology, 218 (3-4), 315-338.

NOMADE S., GAUThier A., GUILlOU H. \& PASTRE J.-F., $2010-{ }^{40} \mathrm{Ar} /{ }^{39} \mathrm{Ar}$ temporal framework for the Alleret maar lacustrine sequence (French Massif-Central): volcanological and paleoclimatic implications. Quaternary Geochronology, 5 (1), 20-27.

NOMADE S., SCAILlET S., PASTRE J.-F. \& NEHLIG P. 2012 - Pyroclastic chronology of the Sancy stratovolcano (MontDore, French Massif Central): New high-precision ${ }^{40} \mathrm{Ar} /{ }^{39} \mathrm{Ar}$ constraints. Journal of Volcanology and Geothermal Research, 225-226, 1-12.

PALOMBO M.R, RAIA P. \& GIOVINAZZO C., 2005 - Structural changes in mammalian communities of Italian peninsula. In M.J. Head \& P.L. Gibbard (eds.), Early-Middle Pleistocene-Transitions: The Land-Ocean Evidence. Special Publication - Geological Society of London, 247. The Geological Society, London \& AAPG Bookstore, Tulsa, 251-262.

PARFENOFF A., POMEROL C. \& TOURENQ J., 1970 - Les minéraux en grains, méthodes d'étude et de détermination. Masson, Paris, $578 \mathrm{p}$

PASTRE J.-F. \& CANTAGREL J.-M., 2001 - Téphrostratigraphie du Mont-Dore (Massif central français). Quaternaire, 12 (4), 249-267.

PASTRE J.-F., GAUTHIER A., NOMADE S., ORTH P., ANDRIEU A., GOUPILLE F., GUILLOU H., KUNESCH S., SCAILLET S. \& RENNE P.R., 2007 - The Alleret maar (Massif Central, France) a new lacustrine sequence of the early Middle Pleistocene in western Europe. Comptes Rendus Géosciences, 339 (16), 987-997.

POPESCU S.-M., BILTEKIN D., WINTER H., SUC J-P., MELINTE-DOBRINESCU M.C., KLOTZ S., RABINEAU M., COMBOURIEU-NEBOUT N., CLAUZON G. \& DEACONU F., 2010 - Pliocene and Lower Pleistocene vegetation and climate changes at the European scale: Long pollen records and climatostratigraphy. Quaternary International, 219 (1-2), 152-167.
PROKOPENKO A.A., HINNOV L.A., WILLIAMS D.F. \& KUZMIN M.I., 2006 - Orbital forcing of continental climate during the Pleistocene: a complete astronomically tuned climatic record from Lake Baikal, SE Siberia. Quaternary Science Reviews, 25 (23/24), 3431-3457.

REILLE M. \& DE BEAULIEU J.-L., 1990 - Pollen analysis of a long upper Pleistocene continental sequence in a Velay maar (Massif Central, France). Palaeogeography, Palaeoclimatology, Palaeoeco$\log y, \mathbf{8 0}(1), 35-48$

REILLE M. \& DE BEAULIEU J.-L., 1995 - Long Pleistocene pollen records from the Praclaux Crater, South-Central France. Quaternary Research, 44 (2), 205-215.

REILLE M., ANDRIEU V., BEAULIEU J.-L. DE, GUENET P. \& GOEURY C., 1998 - A long pollen record from Lac du Bouchet, Massif central, France: for the period ca. 325 to $100 \mathrm{ka} \mathrm{BP}$ (OIS 9c to OIS 5e). Quaternary Science Reviews, 17 (12), 1107-1123.

REILLE M., BEAULIEU J.-L. DE, SVOBODOVA H., ANDRIEUPONEL V. \& GOEURY C., 2000 - Pollen analytical biostratigraphy of the last five climatic sequence from the Velay region (Massif Central, France). Journal of Quaternary Science, 15 (7), 665-685.

RENNE P.R., KARNER D.B. \& LUDWIG K.R., 1998 - Absolute ages aren't exact. Science, 282 (5395), 1840-1841.

ROGER S., FÉRAUD G., DE BEAULIEU J.-L., THOUVENY N., COULON C., COCHEMÉ J.J, ANDRIEU V \& WILLIAMS T., $1999-{ }^{40} \mathrm{Ar} /{ }^{39} \mathrm{Ar}$ dating on tephra of the Velay maars (France): implications for the Late Pleistocene proxy-cimatic record. Earth and Planetary Science Letters, 170 (3), 287-299.

RUDDIMAN W.F., 2003 - Orbital forcing ice volume and greenhouse gases. Quaternary Science Reviews, 22 (15-17), 1597-1629.

SARTORI M., EVANS M.E., HELLER F., TSATSKIN A. \& HAN J.M., 2005 - The last glacial/interglacial cycle at two sites in the Chinese Loess Plateau: Mineral magnetic, grain-size and ${ }^{10} \mathrm{Be}$ measurements and estimates of palaeoprecipitation. Palaeogeography, Palaeoclimatology, Palaeoecology, 222 (1-2), 145-160.

SCAILLET S.,VITA-SCAILLET G. \& GUILLOU H., 2008 - Oldest human footprints dated by Ar/Ar. Earth and Planetary Science Letters, 275 (3-4), 320-325.

STEIGER R. H. \& JÄGER E. 1977 - Subcommission on geochronology: convention on the use of decay constants in geo- and cosmochronology. Earth and Planetary Science Letters, 36 (3), 359-362.

THOUVENY N., CREER K.M. \& BLUNK I., 1990 - Extension of the Lac du Bouchet palaeomagnetic record over the last 120,000 years. Earth and Planetary Science Letters, 97 (1-2), 140-161.

THOUVENY N., DE BEAULIEU J.-L., BONIFAY E., CREER K.M. GUIOT J., ICOLE M., JOHNSEN S., JOUZEL J., REILLE M. WILLIAMS T. \& WILLIAMSON D., 1994 - Climate variations in Europe over the past $140 \mathrm{kyr}$ deduced from rock magnetism. Nature, 371 (6497), 503-506

TRUZE E. \& KELTS K., 1993 - Sedimentology and paleoenvironment from the maar Lac du Bouchet for the last climatic cycle, 0-120,000 years (Massif Central, France). In J.F.W. Negedank \& B. Zolitschka (eds.), Paleolimnology of European maar lakes. Lecture Notes in Earth Sciences, 49. Springer-Verlag, Berlin \& New York, 237-275.

TZEDAKIS P.C., HOOGHIEMSTRA H. \& PÄLIKE H., 2006 - The last 1.35 million years at Tenaghi Philippon: revised chronostratigraphy and long-term vegetation trends. Quaternary Science Reviews, 25 (23-24), 3416-3430.

VALADAS B., 1983 - Les hautes terres du Massif Central français. Contribution à l'étude des morphodynamiques récentes sur versants cristallins et volcaniques. Thèse de Doctorat d'Etat, Université Paris 1 Panthéon-Sorbonne, Paris, $927 \mathrm{p}$.

VENZ K., HODELL D.A, STANTON C. \& WARNKE D.A., 1999 - A 1.0 Myr record of Glacial North Atlantic Intermediate Water variability from ODP site 982 in the northeast Atlantic. Paleoceanography, 14 (1), 42-52.

ZACHOS J., PAGANI M., SLOAN L., THOMAS E. \& BILLUPS K., 2001 - Trends, Rhythms, and Aberrations in Global Climate $65 \mathrm{Ma}$ to Present. Science, 292 (5517), 686-693. 